\title{
Methods in Ranking Fuzzy Numbers: A Unified Index and Comparative Reviews
}

\author{
Thanh-Lam Nguyen \\ Office of Scientific Research, Lac Hong University, Dong Nai, Vietnam \\ Correspondence should be addressed to Thanh-Lam Nguyen; green4rest.vn@gmail.com
}

Received 6 April 2017; Revised 30 May 2017; Accepted 7 June 2017; Published 13 July 2017

Academic Editor: Omar Abu Arqub

Copyright (c) 2017 Thanh-Lam Nguyen. This is an open access article distributed under the Creative Commons Attribution License, which permits unrestricted use, distribution, and reproduction in any medium, provided the original work is properly cited.

\begin{abstract}
Fuzzy set theory, extensively applied in abundant disciplines, has been recognized as a plausible tool in dealing with uncertain and vague information due to its prowess in mathematically manipulating the knowledge of imprecision. In fuzzy-data comparisons, exploring the general ranking measure that is capable of consistently differentiating the magnitude of fuzzy numbers has widely captivated academics' attention. To date, numerous indices have been established; however, counterintuition, less discrimination, and/or inconsistency on their fuzzy-number rating outcomes have prohibited their comprehensive implementation. To ameliorate their manifested ranking weaknesses, this paper proposes a unified index that multiplies weighted-mean and weighted-area discriminatory components of a fuzzy number, respectively, called centroid value and attitude-incorporated left-and-right area. From theoretical proof of consistency property and comparative studies for triangular, triangular-and-trapezoidal mixed, and nonlinear fuzzy numbers, the unified index demonstrates conspicuous ranking gains in terms of intuition support, consistency, reliability, and computational simplicity capability. More importantly, the unified index possesses the consistency property for ranking fuzzy numbers and their images as well as for symmetric fuzzy numbers with an identical altitude which is a rather critical property for accurate matching and/or retrieval of information in the field of computer vision and image pattern recognition.
\end{abstract}

\section{Introduction}

It has been well recognized that uncertainty inevitably exists in several real-world phenomena due to the inherent errors or impreciseness of measurement tools, methods, and uncontrollable conditions $[1,2]$. In managing the uncertainty and vagueness, the fuzzy set theory has been widely considered as a powerful tool $[3,4]$. And many scholars have made special efforts in proposing more and more effective approaches to deal with practical problems in the fuzzy environment. Since the inception of the fuzzy set theory, Soliman and Mantawy [5] showed that five major strongly connected branches have been developed, including fuzzy mathematics, fuzzy logic and artificial intelligence, fuzzy systems, uncertainty and information, and fuzzy decision-making. Their subbranches have also been established; for example, fuzzy differential equations [6-14] and fuzzy integrodifferential equations [1522] are of fuzzy mathematics while fuzzy-number ranking, the focus of this paper, is of fuzzy decision-making. Specifically, based on its feasible mathematical capacity for representing the imprecise information in practice, we have observed many successful cases spreading in disparate disciplines, such as robot selection [23], supplier selection [24], logistics center allocation [25], facility location determination [26], choosing mining methods [27], manufacturing process monitoring $[1,2,28-31]$, cutting force prediction [32], firm-environmental knowledge management [33, 34], green supply-chain operation [35], and weapon procurement decision [36]. Apparently, to find their best alternative, those decisive problems are evaluated under resource constraints and with to some extent linguistic preference of multiattribute, which is realized from users' perspectives, as well as subjective quantification of multiple characteristics, which is assessed from decision-makers [2, 3, 37-39]. In these cases, fuzzy-data comparisons and rankings are inevitable.

As the fuzzy data (fuzzy numbers) can overlap with each other and are represented by possibility distributions, their comparison and ordering, not akin to that of real numbers which can be linearly ordered, become challenging and cumbersome. Generally, to rank fuzzy quantities, a set of 
fuzzy numbers, through a specific defuzzification measure, is converted into real numbers, where a natural order between them is definitive [40]. However, even when ordering for a set of single fuzzy numbers, this defuzzification procedure does lose a certain amount of fuzziness/imprecision information existing in the original data [1,40-47], not to mention the ordering for problems of multicriteria decision-making, where sets of fuzzy numbers have experienced some mathematical operations [48]; therefore, much endeavor has been attempted to minimize loss of information, a fundamental problem for fuzzy-data analysis.

Jain [49] in 1977 first launched a fuzzy set rating procedure for multiple-aspect decision-making. Since then, exploring a general ranking measure, capable of consistently differentiating the magnitude of fuzzy numbers, has widely captivated academics' attention [50]. Nowadays, a majority of diverse improved approaches/indices established from widerange perspectives focus on either compensating their predecessors' failures in certain reasonable properties for ordering of fuzzy quantities $[43,44]$ or resolving the counterintuitive, indiscriminate, and/or inconsistent rating outcomes among certain types of fuzzy numbers [42, 51-54].

In general, the existing ranking measures can be classified into two main categories:

(i) Indices that value the fuzzy number itself such as center-, area-, and deviation-driven ordering measures

(ii) Indices that not only evaluate the fuzzy number itself, but also gauge decision-maker's attitude in regard to specific purposes such as confidence and risk

In category one, Yager [55] and Lee and Li [56] first borrowed statistical center-oriented measures for assessing fuzzy numbers, where the former constructed a centroid (weighted mean) index and the latter developed mean and standard deviation indices; however, Cheng [57] pointed out their inefficient manipulation of the fuzzy numbers that possesses unusually large or small data (outliers) and mean-and-spread values. To cope with the inefficiencies, R. Saneifard and R. Saneifard [58], Zhang et al. [59], Bodjanova [60, 61], and Yamashiro [62] suggested a median index, a resistant measure of the center, to take into account data located on the tails; Cheng [57] proposed coefficient-of-variation and distance indices; but both indices were later criticized for some inconsistent ordering among specific types of fuzzy numbers [63]. Based on the area between the centroid point and the original point, Chu and Tsao [63] succeeded in establishing an areadriven ranking index; unfortunately, because of its inherent computation flaw, the area index was questioned by Wang and Lee [64] who illustrated some numerical examples to show its counterintuitive results and further provided a compelling revised index to resolve the problem. Nonetheless, Wang and Lee's area index does have its own deficiency of ordering correctness when encountering fuzzy numbers with identical centroid points [65]. By defining fuzzy-number maximal and minimal reference sets, Wang et al. [66] first introduced a deviation-driven ordering index by combining right-and-left deviation degree with the coefficient of relative variation; not surprisingly, this index was argued (1) bearing mathematical incapability with zero value in the denominator [53] and pointed out (2) leaving substantial room for improvement under some special occasions such as fuzzy numbers with the same left, right, and total utilities [39] as well as ranking fuzzy numbers' images [46].

Emphatically, the aforementioned drawbacks plagued on this deviation-driven ordering index have somewhat reignited the development of category two, initially proposed by Liou and Wang [67] in 1992, and contrived ranking measures that not only evaluate the fuzzy number itself, but also consider decision-maker's attitude in relation to specific purposes. The evidence can be seen in the most recent works; for example, to remove shortages of Wang et al's deviation-degree index [66], Wang and Luo [39] incorporated decision-maker's attitude towards risk into left-and-right area between fuzzynumber points and the positive-and-negative ideal points; to improve Liou and Wang's index [67], Yu and Dat [48] incorporated decision-maker's attitude regarding confidence into left-right-total integral value subjected to fuzzy-number median value. More recently, Das and Guha [68] proposed a new ranking approach by computing the centroid point of trapezoidal intuitionistic fuzzy numbers (TrIFN) and applied it to solve multicriteria decision-making problems in combination with expert's degree of satisfaction. However, their formulas fail to effectively work when their TrIFN $(a, b, c, d)$ becomes either $(a, a, c, d)$ or $(a, b, c, c)$ or the satisfaction/dissatisfaction degree takes a value of zero. In addition, as shown in Table 1, certain shortcomings such as counterintuition, less reliability, inconsistency, complex/laborious computation, and indecisive ranking results have been found to be existing in several current ranking approaches.

Ostensibly, as opposed to the prolific ranking indices to date that have been presented in category one, the established ranking indices related to category two are still few, leaving a wide range of topics for further investigation. Based on the integration of the two categories, this paper proposes a unified index that multiplies weighted mean and weighted area, two discriminatory components of a fuzzy number, respectively, called centroid value (the category one measurement) and attitude-incorporated left-and-right area (the category two measurement). According to comprehensively comparative studies from triangular, triangular-andtrapezoidal mixed, and nonlinear fuzzy numbers, the unified index demonstrates obtrusive ranking benefits with respect to intuition support, computational easiness, consistency, and reliability capability.

Aside from the Introduction, the remainder of this paper is organized into four sections as follows. Section 2 provides preliminary definitions and remarks for the research. The proposed unified index is described in Section 3, whose comparative studies with some existing ranking indices are done with several literature-exemplary fuzzy numbers in Section 4 . Summary and conclusions make up the last section.

\section{Preliminaries}

The following definitions and remarks are mainly adopted from Zimmermann [69] and Lee [70]. 
Definition 1 (fuzzy subset). Let $\mathbb{R}$ be a nonempty set. The fuzzy subset $\widetilde{A}$ of $\mathbb{R}$ is defined by a function $\xi_{\widetilde{A}}: \mathbb{R} \rightarrow[0,1]$. $\xi_{\widetilde{A}}$ is called a membership function.

Definition 2 ( $\alpha$-cut set). The $\alpha$-cut set of $\widetilde{A}$, denoted by $\widetilde{A}_{\alpha c}$, is defined by $\widetilde{A}_{\alpha c}=\left\{x \in \mathbb{R}: \xi_{\widetilde{A}}(x) \geq \alpha\right\}$ for all $\alpha \in(0,1]$. The 0 -cut set $\widetilde{A}_{0 c}$ is defined as the closure of the set $\{x \in \mathbb{R}$ : $\left.\xi_{\widetilde{A}}(x)>0\right\}$.

Definition 3 ( $\alpha$-level set). The $\alpha$-level set of $\widetilde{A}$, denoted by $\widetilde{A}_{\alpha}$, is defined by $\widetilde{A}_{\alpha}=\left\{x \in \mathbb{R}: \xi_{\widetilde{A}}(x)=\alpha\right\}$ for all $\alpha \in[0,1]$.

Definition 4 (fuzzy number). A fuzzy number $\widetilde{A}=$ $(a, b, c, d ; w)$ is described as any fuzzy subset of the real line $\mathbb{R}$ with the membership function $\xi_{\widetilde{A}}(x)$ which is given by

$$
\xi_{\widetilde{A}}(x)= \begin{cases}\xi_{\widetilde{A}}^{L}(x), & a \leq x<b \\ w, & b \leq x \leq c \\ \xi_{\widetilde{A}}^{R}(x), & c<x \leq d \\ 0, & \text { otherwise }\end{cases}
$$

where $0 \leq w \leq 1$ is a constant and $\xi_{\widetilde{A}}^{L}(x), \xi_{\widetilde{A}}^{R}(x)$ are continuous functions on $[0,1]$.

A fuzzy number has the following properties:

(i) $\widetilde{A}$ is normal if there exists an $x \in \mathbb{R}$ such that $\xi_{\widetilde{A}}(x)=$ 1 ; that is, $w=1$.

(ii) $\xi_{\widetilde{A}}(x)$ is fuzzy convex; that is, $\xi_{\widetilde{A}}(t x+(1-t) y) \geq$ $\min \left\{\xi_{\widetilde{A}}(x), \xi_{\widetilde{A}}(y)\right\}$ for $t \in[0,1]$.

(iii) $\xi_{\widetilde{A}}(x)$ is upper semicontinuous; that is, $\{x \in \mathbb{R}$ : $\left.\xi_{\widetilde{A}}(x) \geq \alpha\right\}$ is a closed subset of $\mathbb{R}$ for each $\alpha \in(0,1]$.

(iv) The 0-level set $\widetilde{A}_{0}$ is a closed and bounded subset of $\mathbb{R}$.

Since $\widetilde{A}_{\alpha} \subset \widetilde{A}_{0}$ for each $\alpha \in(0,1]$, condition (iv) shows that the $\alpha$-level sets $\widetilde{A}_{\alpha}$ are bounded subsets of $\mathbb{R}$ for all $\alpha \in(0,1]$. It is well known that condition (ii) is satisfied if and only if the $\alpha$-level set $\widetilde{A}_{\alpha}$ is a convex subset of $\mathbb{R}$. Therefore, from conditions (i)-(iv), it is implied that if $\widetilde{A}$ is a fuzzy number, then the $\alpha$-level set of $\widetilde{A}$ is a closed, bounded, and convex subset of $\mathbb{R}$, that is, a closed interval in $\mathbb{R}$, denoted by $\widetilde{A}_{\alpha}=\left[\widetilde{A}_{\alpha}^{L}, \widetilde{A}_{\alpha}^{U}\right]$.

Remark 5. Let $\widetilde{A}$ be a fuzzy number. Then, the following statements hold true:

(i) $\widetilde{A}_{\alpha}^{L} \leq \widetilde{A}_{\alpha}^{U}$ for all $\alpha \in[0,1]$.

(ii) $\widetilde{A}_{\alpha}^{L}$ is increasing with respect to $\alpha \in[0,1]$; that is, $\widetilde{A}_{\alpha}^{L} \leq \widetilde{A}_{\beta}^{L}$ for $0 \leq \alpha<\beta \leq 1$.

(iii) $\widetilde{A}_{\alpha}^{U}$ is decreasing with respect to $\alpha \in[0,1]$; that is, $\widetilde{A}_{\alpha}^{U} \geq \widetilde{A}_{\beta}^{U}$ for $0 \leq \alpha<\beta \leq 1$.

Remark 6. Let $\widetilde{A}$ be a fuzzy number such that its membership function is strictly increasing on interval $[a, b]$ and strictly

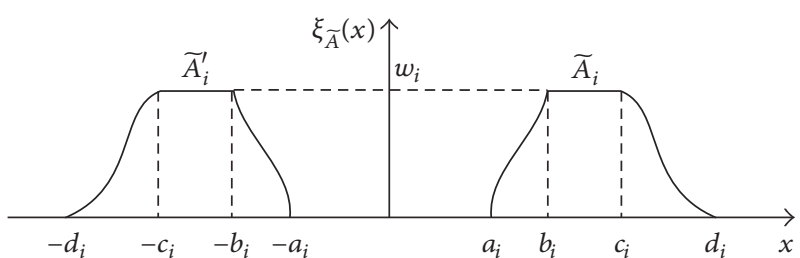

Figure 1: $\widetilde{A}_{i}$ is the image of $\widetilde{A}_{i}$.

decreasing on interval $[c, d]$. From the fact of strict monotonicity, $\xi_{\widetilde{A}}^{L}(x)$ and $\xi_{\widetilde{A}}^{R}(x)$ are continuous functions on $[0,1]$. This implies that $\widetilde{A}$ is also a real fuzzy number.

Definition 7 (the image of a fuzzy number [4]). Let $n$ fuzzy numbers be $\widetilde{A}_{i}=\left(a_{i}, b_{i}, c_{i}, d_{i} ; w_{i}\right)(i=\overline{1, n})$. Then, the image of $\widetilde{A}_{i}$ is $\widetilde{A}_{i}^{\prime}=\left(-d_{i},-c_{i},-b_{i},-a_{i} ; w_{i}\right)$, as shown in Figure 1.

\section{A Unified Index}

Based on integration of the two aforementioned categories for ranking fuzzy numbers, a unified index, which combines centroid value (weighted mean) and attitude-incorporated leftand-right area (weighted area), is proposed in this section.

Definition 8 (centroid value (a center-driven measure that belongs to category one)). Centroid value of a fuzzy number $\widetilde{A}_{i}=\left(a_{i}, b_{i}, c_{i}, d_{i} ; w_{i}\right)$ for $i=\overline{1, n}$, symbolized by $\mathrm{CV}_{i}$, is defined as $[3,4,38,63,65,71]$

$$
\mathrm{CV}_{i}=\frac{\int_{a_{i}}^{d_{i}} x \xi_{\widetilde{A}_{i}}(x) d x}{\int_{a_{i}}^{d_{i}} \xi_{\widetilde{A}_{i}}(x) d x}
$$

From the statistical point of view, it is the weighted mean of $\widetilde{A}_{i}$, meaning that when $\widetilde{A}_{i}=\left(a, a, a, a ; w_{i}\right)$, we can accordingly have $\mathrm{CV}_{i}=a$.

Definition 9 (left-and-right areas (an area-driven measure that belongs to category one)). Left-and-right areas of a fuzzy number $\widetilde{A}_{i}$ for $i=\overline{1, n}$, denoted by $S_{i}^{L}$ and $S_{i}^{R}$, are given by

$$
\begin{aligned}
& S_{i}^{L}=\left|\int_{0}^{w_{i}} g_{\widetilde{A}_{i}}^{L}(y) d y\right|, \\
& S_{i}^{R}=\left|\int_{0}^{w_{i}} g_{\widetilde{A}_{i}}^{R}(y) d y\right|,
\end{aligned}
$$

where $g_{\widetilde{A}_{i}}^{L}(y)$ and $g_{\widetilde{A}_{i}}^{R}(y)$ stand for inverse functions of the left-and-right membership functions, $\xi_{\widetilde{A}_{i}}^{L}(x)$ and $\xi_{\widetilde{A}_{i}}^{R}(x)$, respectively, and visual views of $S_{i}^{L}$ and $S_{i}^{R}$ are shown in Figure 2 [72].

Now, a fuzzy-number measure belonging to category two is presented. It also contemplates decision-maker's attitude as regards data revelation, called attitude-incorporated left-andright area, signified by $\mathrm{AA}_{i}^{\lambda}$.

$$
\mathrm{AA}_{i}^{\lambda}=\lambda S_{i}^{R}+(1-\lambda) S_{i}^{L},
$$



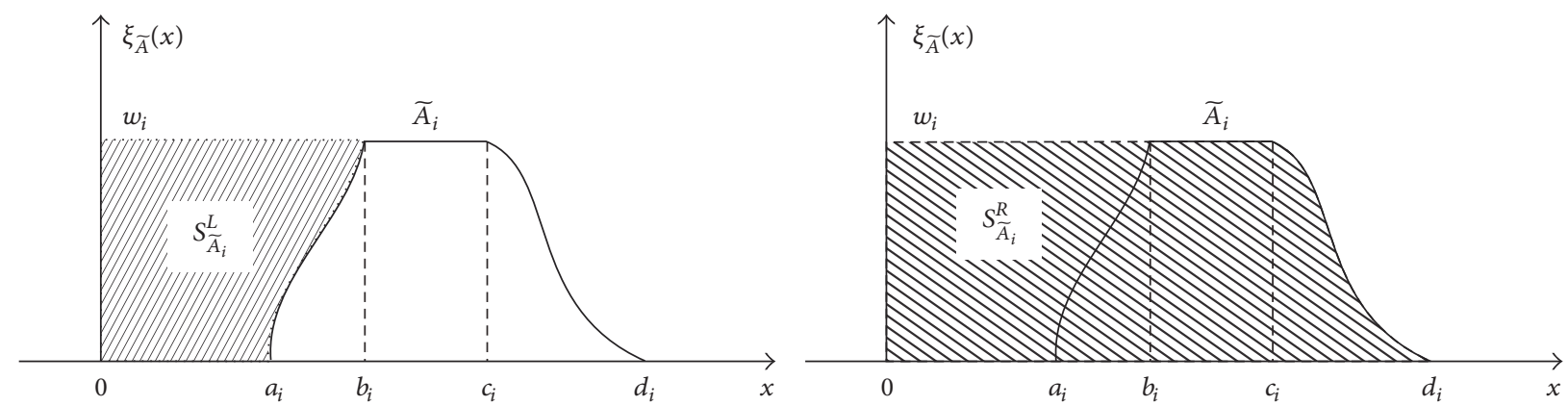

Figure 2: Left area $S_{i}^{L}$ and right area $S_{i}^{R}$.

where $\lambda \in[0,1]$ is level of optimism reflecting a data-revelation optimism degree of a decision-maker, where the larger the $\lambda$ set by the decision-maker is, the more optimistic attitude the decision-maker has on the data revelation. Two extreme cases are $\lambda=0$, meaning the decision-maker is completely pessimistic, and $\lambda=1$, meaning the decisionmaker is completely optimistic. Case $\lambda=1 / 2$ reflects a neutral decision attitude. From the mathematical viewpoint, (4) can be seen as a weighted-area value of $\widetilde{A}_{i}$.

For boosting the fuzzy-number discrimination power, let us consider an index named $\mathrm{UI}_{i}^{\lambda}$ by multiplying two sizediscriminatory values of a fuzzy number; that is,

$$
\mathrm{UI}_{i}^{\lambda}=\left(\mathrm{CV}_{i}+\varepsilon_{i}\right)\left[\lambda S_{i}^{R}+(1-\lambda) S_{i}^{L}\right] \text {. }
$$

$\mathrm{UI}_{i}^{\lambda}$ is called unified index. And, $\varepsilon_{i}$ initially takes a very small real number which is quantifiable and rational for comparing the targeted fuzzy numbers whose centroid values take a value of zero, $\mathrm{CV}_{i}=0$. It is used to provide consistent ranking power when $\mathrm{CV}_{i}=0$. Particularly, this paper suggests using $\varepsilon_{i}=w_{i} \times 10^{-9}$ so that we can efficiently rank fuzzy numbers that have similar centroids but different height.

Remark 10. Consider the ranking of two fuzzy numbers, $\widetilde{A}_{i}$ and $\widetilde{A}_{j}$. Given the data-optimistic level $\lambda$, from (5), we obtain their realized unified indices, $\mathrm{UI}_{i}^{\lambda}$ and $\mathrm{UI}_{j}^{\lambda}$. Then, the following decisions can be made:

(i) At the data-optimistic level $\lambda$, if $\mathrm{UI}_{i}^{\lambda}>\mathrm{UI}_{j}^{\lambda}$, then ${\widetilde{A_{i}}}_{i}>$ $\widetilde{A}_{j}$.

(ii) At the data-optimistic level $\lambda$, if $\mathrm{UI}_{i}^{\lambda}<\mathrm{UI}_{j}^{\lambda}$, then $\widetilde{A}_{i} \prec$ $\widetilde{A}_{j}$.

(iii) At the data-optimistic level $\lambda$, if $\mathrm{UI}_{i}^{\lambda}=\mathrm{UI}_{j}^{\lambda}$, then $\widetilde{A}_{i} \simeq$ $\widetilde{A}_{j}$.

Now, we will prove the unified index's consistency property when ranking fuzzy numbers and their images. Without loss of generality, $\mathrm{CV}_{i} \neq 0$ is considered in the following.

Proposition 11. Let $\widetilde{A}_{i}^{\prime}=\left(-d_{i},-c_{i},-b_{i},-a_{i} ; w_{i}\right)$ be the image of a fuzzy number $\widetilde{A}_{i}=\left(a_{i}, b_{i}, c_{i}, d_{i} ; w_{i}\right)$ for $i=\overline{1, n}$. Its centroid value is $C V_{i^{\prime}}=-C V_{i}$, left-and-right areas are $S_{i^{\prime}}^{R}=S_{i}^{L}$ and $S_{i^{\prime}}^{L}=$ $S_{i}^{R}$, attitude-incorporated left-and-right area is $A A_{i^{\prime}}^{\lambda}=A A_{i}^{1-\lambda}$ and $A A_{i^{\prime}}^{1-\lambda}=A A_{i}^{\lambda}$, and unified index is $U I_{i^{\prime}}^{\lambda}=-U I_{i}^{1-\lambda}$ and $U I_{i^{\prime}}^{1-\lambda}=-U I_{i}^{\lambda}$.

Proof. From (2),

$$
\mathrm{CV}_{i^{\prime}}=\frac{\int_{-d_{i}}^{-a_{i}} x \xi_{\widetilde{A}_{i}^{\prime}}(x) d x}{\int_{-d_{i}}^{-a_{i}} \xi_{\widetilde{A}_{i}^{\prime}}(x) d x}=-\frac{\int_{a_{i}}^{d_{i}} x \xi_{\widetilde{A}_{i}}(x) d x}{\int_{a_{i}}^{d_{i}} \xi_{\widetilde{A}_{i}}(x) d x}=-\mathrm{CV}_{i}
$$

Based on (3),

$$
\begin{aligned}
& S_{i^{\prime}}^{L}=\left|\int_{0}^{w_{i}} g_{\widetilde{A}_{i}^{\prime}}^{L}(y) d y\right|=\left|\int_{0}^{w_{i}} g_{\widetilde{A}_{i}}^{R}(y) d y\right|=S_{i}^{R} \\
& S_{i^{\prime}}^{R}=\left|\int_{0}^{w_{i}} g_{\widetilde{A}_{i}^{\prime}}^{R}(y) d y\right|=\left|\int_{0}^{w_{i}} g_{\widetilde{A}_{i}}^{L}(y) d y\right|=S_{i}^{L} .
\end{aligned}
$$

According to (4) and with the above results, $S_{i^{\prime}}^{R}=S_{i}^{L}$ and $S_{i^{\prime}}^{L}=$ $S_{i}^{R}$, we further have

$$
\mathrm{AA}_{i^{\prime}}^{\lambda}=\lambda S_{i^{\prime}}^{R}+(1-\lambda) S_{i^{\prime}}^{L}=\lambda S_{i}^{L}+(1-\lambda) S_{i}^{R}=\mathrm{AA}_{i}^{1-\lambda} .
$$

Similarly,

$$
\mathrm{AA}_{i^{\prime}}^{1-\lambda}=\left(1-\lambda S_{i^{\prime}}^{R}\right)+\lambda S_{i^{\prime}}^{L}=(1-\lambda) S_{i}^{L}+\lambda S_{i}^{R}=\mathrm{AA}_{i}^{\lambda} .
$$

Finally, regarding (5) and the aforementioned outcomes, we can simply obtain

$$
\begin{aligned}
\mathrm{UI}_{i^{\prime}}^{\lambda} & =\mathrm{CV}_{i^{\prime}}\left[\lambda S_{i^{\prime}}^{R}+(1-\lambda) S_{i^{\prime}}^{L}\right]=-\mathrm{UI}_{i}^{1-\lambda}, \\
\mathrm{UI}_{i^{\prime}}^{1-\lambda} & =\mathrm{CV}_{i^{\prime}}\left[(1-\lambda) S_{i^{\prime}}^{R}+\lambda S_{i^{\prime}}^{L}\right]=-\mathrm{UI}_{i}^{\lambda} .
\end{aligned}
$$

We complete the proof.

Proposition 12. Let a set of fuzzy numbers be $\widetilde{A}_{k}=\left(a_{k}, b_{k}\right.$, $\left.c_{k}, d_{k} ; w_{k}\right)$ and their images $\widetilde{A}_{k}=\left(-d_{k},-c_{k},-b_{k},-a_{k} ; w_{k}\right), k=$ $\overline{1, n}$. For a pairwise comparison of $\widetilde{A}_{i}$ and $\widetilde{A}_{j}$ for $i, j \in k$, two statements hold true: (1) $U I_{i}^{\lambda}>U I_{j}^{\lambda}$ if and only if $U I_{i^{\prime}}^{1-\lambda}<U I_{j^{\prime}}^{1-\lambda}$ and (2) $U I_{i}^{\lambda}<U I_{j}^{\lambda}$ if and only if $U I_{i^{\prime}}^{1-\lambda}>U I_{j^{\prime}}^{1-\lambda}$. 
Proof. Consider $\mathrm{UI}_{i}^{\lambda}>\mathrm{UI}_{j}^{\lambda}$. From Proposition 11, we have the results $\mathrm{UI}_{i}^{\lambda}=-\mathrm{UI}_{i^{\prime}}^{1-\lambda}$ and $\mathrm{UI}_{j}^{\lambda}=-\mathrm{UI}_{j^{\prime}}^{1-\lambda}$. Thus, $\mathrm{UI}_{i^{\prime}}^{1-\lambda}<$ $\mathrm{UI}_{j^{\prime}}^{1-\lambda}$. On the other hand, consider $\mathrm{UI}_{i^{\prime}}^{1-\lambda}<\mathrm{UI}_{j^{\prime}}^{1-\lambda}$. According to Proposition $11, \mathrm{UI}_{i^{\prime}}^{1-\lambda}=-\mathrm{UI}_{i}^{\lambda}$ and $\mathrm{UI}_{j^{\prime}}^{1-\lambda}=-\mathrm{UI}_{j}^{\lambda}$. Hence, $\mathrm{UI}_{i}^{\lambda}>\mathrm{UI}_{j}^{\lambda}$. Overall, the proof is completed.

Remark 13. Let a set of fuzzy numbers be $\widetilde{A}_{k}=\left(a_{k}, b_{k}, c_{k}\right.$, $\left.d_{k} ; w_{k}\right)$ and their images $\widetilde{A}_{k}=\left(-d_{k},-c_{k},-b_{k},-a_{k} ; w_{k}\right), k=$ $\overline{1, n}$. As regards Remark 10 and Propositions 11 and 12, the following decisions can be made for a pairwise comparison of $\widetilde{A}_{i}$ and $\widetilde{A}_{j}$, for $i, j \in k$.

(i) At the data-optimistic level $\lambda$, if $\mathrm{UI}_{i}^{\lambda}>\mathrm{UI}_{j}^{\lambda}$, which is equivalent to $\mathrm{UI}_{i^{\prime}}^{1-\lambda}<\mathrm{UI}_{j^{\prime}}^{1-\lambda}$, then $\widetilde{A}_{i}>\widetilde{A}_{j}$, which is equivalent to $\widetilde{A}_{i}^{\prime}<\widetilde{A}_{j}^{\prime}$.

(ii) At the data-optimistic level $\lambda$, if $\mathrm{UI}_{i}^{\lambda}<\mathrm{UI}_{j}^{\lambda}$, which is equivalent to $\mathrm{UI}_{i^{\prime}}^{1-\lambda}>\mathrm{UI}_{j^{\prime}}^{1-\lambda}$, then $\widetilde{A}_{i} \prec \widetilde{A}_{j}$, which is equivalent to $\widetilde{A}_{i}^{\prime}>\widetilde{A}_{j}$.

(iii) At the data-optimistic level $\lambda$, if $\mathrm{UI}_{i}^{\lambda}=\mathrm{UI}_{j}^{\lambda}$, which is equivalent to $\mathrm{UI}_{i^{\prime}}^{1-\lambda}=\mathrm{UI}_{j^{\prime}}^{1-\lambda}$, then $\widetilde{A}_{i} \simeq \widetilde{A}_{j}$, which is equivalent to $\vec{A}_{i} \simeq \vec{A}_{j}$.

Finally, the following theory is very useful for ranking "symmetric" fuzzy numbers with an identical altitude.

Theorem 14. Consider a set of "symmetric" fuzzy numbers, $\widetilde{A}_{k}=\left(a_{k}, b_{k}, c_{k}, d_{k} ; w_{k}\right)$, and their images $\widetilde{A}_{k}^{\prime}=\left(-d_{k},-c_{k},-b_{k}\right.$, $\left.-a_{k} ; w_{k}\right), k=\overline{1, n}$. By using the unified index, the pairwise comparison of $\widetilde{A}_{i}$ and $\widetilde{A}_{j}$ for $i, j \in k$ is $\lambda=0.5, \widetilde{A}_{i} \simeq \widetilde{A}_{j}\left(\widetilde{A}_{i} \simeq\right.$ $\left.\widetilde{A}_{j}^{\prime}\right), \lambda \in[0,0.5), \widetilde{A}_{i} \prec \widetilde{A}_{j}\left(\widetilde{A}_{i}^{\prime}>\widetilde{A}_{j}^{\prime}\right)$, and $\lambda \in(0.5,1]$, $\widetilde{A}_{i}>\widetilde{A}_{j}\left(\widetilde{A}_{i}^{\prime} \prec \widetilde{A}_{j}^{\prime}\right)$.

Proof. (i) Since $\widetilde{A}_{i}=\left(a_{i}, b_{i}, c_{i}, d_{i} ; w\right)$ and $\widetilde{A}_{j}=\left(a_{j}, b_{j}, c_{j}\right.$, $\left.d_{j} ; w\right)$ for $i, j=\overline{1, n}$ are symmetric, we have $a_{i}+d_{i}=a_{j}+d_{j}$. Moreover, from (2),

$$
\begin{aligned}
\mathrm{CV}_{i} & =\frac{\int_{a_{i}}^{d_{i}} x \xi_{\widetilde{A}_{i}}(x) d x}{\int_{a_{i}}^{d_{i}} \xi_{\widetilde{A}_{i}}(x) d x}=\frac{a_{i}+d_{i}}{2}, \\
\mathrm{CV}_{j} & =\frac{\int_{a_{j}}^{d_{j}} x \xi_{\widetilde{A}_{j}}(x) d x}{\int_{a_{j}}^{d_{j}} \xi_{\widetilde{A}_{j}}(x) d x}=\frac{a_{j}+d_{j}}{2} .
\end{aligned}
$$

Therefore, $\mathrm{CV}_{i}=\mathrm{CV}_{j}$.

(ii) According to (3) and (4), we have

$$
\begin{aligned}
& \mathrm{AA}_{i}^{\lambda}=\lambda S_{i}^{R}+(1-\lambda) S_{i}^{L} \\
& \mathrm{AA}_{j}^{\lambda}=\lambda S_{j}^{R}+(1-\lambda) S_{j}^{L},
\end{aligned}
$$

where

$$
\begin{array}{r}
S_{i}^{L}=\left|\int_{0}^{w} g_{\widetilde{A}_{i}}^{L}(y) d y\right|, \\
S_{i}^{R}=\left|\int_{0}^{w} g_{\widetilde{A}_{i}}^{R}(y) d y\right| \\
S_{j}^{L}=\left|\int_{0}^{w} g_{\widetilde{A}_{j}}^{L}(y) d y\right|, \\
S_{j}^{R}=\left|\int_{0}^{w} g_{\widetilde{A}_{j}}^{R}(y) d y\right| .
\end{array}
$$

Due to the symmetry, we have $\mathrm{AA}_{i}^{\lambda}<\mathrm{AA}_{j}^{\lambda}$ for $\lambda \epsilon$ $[0,0.5), \mathrm{AA}_{i}^{\lambda}=\mathrm{AA}_{j}^{\lambda}$ when $\lambda=0.5$, and vice versa.

(iii) From (i), (ii), and (5), we have

(i) $\lambda \in[0,0.5), \mathrm{UI}_{i}^{\lambda}<\mathrm{UI}_{j}^{\lambda}$,

(ii) $\lambda=0.5, \mathrm{UI}_{i}^{\lambda}=\mathrm{UI}_{j}^{\lambda}$,

(iii) $\lambda \in(0.5,1], \mathrm{UI}_{i}^{\lambda}>\mathrm{UI}_{j}^{\lambda}$.

Finally, according to Remark 13, we complete the proof.

\section{Comparative Studies}

In this section, several fuzzy-number examples, which are popular in the literature for a wide range of fuzzynumber comparative studies, are used to compare ranking performance between the unified index and some up-todate representative indices from the publications. To make it easier to follow the whole discussion of comparison, Table 1 briefly shows the evaluated types of fuzzy numbers, reference sources, and critical shortcomings of the references. Detailed explanations about performance shortages for existing indices in contrast with the proposed index are subsequently described in Examples 15 22.

It can be noted that, based on Propositions 11 and 12 and Remark 13, the unified index fulfills the consistency property for ranking the fuzzy numbers and their partnered images; for conciseness, in several examples, the consistency of imageranking results is not mentioned or shown on the result tables.

4.1. Ranking of Normal Triangular Fuzzy Numbers. This subsection focuses on the ranking of normal triangular fuzzy numbers with some special shape which are recognizably difficult to discriminate in the literature. First, a case with two congruent fuzzy numbers is employed for checking index's computation easiness; then, the work is extended on three similar fuzzy numbers for contrasting indices' ranking consistency and intuition satisfaction; finally, an example, which includes a slight move-away fuzzy number and two fuzzy numbers with an identical center value and geometric enlargement relationship, is examined with respect to ranking indices' reliability and consistency.

Example 15. Rank two fuzzy numbers $\widetilde{A}_{1}=(1,4,5)$ and $\widetilde{A}_{2}=(2,3,6)$ as shown in Figure 3 [48], which are congruent, 
TABLE 1: The ranking performance assessments for some representative indices as opposed to the unified index.

\begin{tabular}{|c|c|c|c|c|}
\hline Section & Example & Evaluated fuzzy numbers & Compared references & Shortcomings (cf. the index) \\
\hline Section 4.1 & Example 15 & $\begin{array}{l}\widetilde{A}_{1}=(1,4,5) \\
\widetilde{A}_{2}=(2,3,6)\end{array}$ & Yu \& Dat [48] & More laborious in computation \\
\hline Section 4.1 & Example 16 & $\begin{array}{c}\widetilde{A}_{1}=(5,6,7) \\
\widetilde{A}_{2}=(5.9,6,7) \\
\widetilde{A}_{3}=(6,6,7)\end{array}$ & $\begin{array}{c}\text { Chu \& Tsao [63] } \\
\text { Cheng [57] } \\
\text { Yu \& Dat [48] }\end{array}$ & $\begin{array}{l}\text { Counterintuition } \\
\text { Counterintuition } \\
\text { Less reliability }\end{array}$ \\
\hline Section 4.1 & Example 17 & $\begin{array}{l}\widetilde{A}_{1}=(1,3,5) \\
\widetilde{A}_{2}=(2,3,4) \\
\widetilde{A}_{3}=(1,4,6)\end{array}$ & Liou \& Wang [67], Yu \& Dat [48] & $\begin{array}{c}\text { Inconsistency } \\
\text { Counterintuition at } \lambda=0\end{array}$ \\
\hline Section 4.2 & Example 18 & $\begin{array}{l}\widetilde{A_{1}}=(1,5,5) \\
\widetilde{A}_{2}=(2,3,5,5)\end{array}$ & Zhang et al. [73] & $\begin{array}{l}\text { Computation complexity } \\
\text { Inconsistency }\end{array}$ \\
\hline Section 4.2 & Example 19 & $\begin{array}{l}\widetilde{A}_{1}=(0,3,6) \\
\widetilde{A}_{2}=(-1,0,2) \\
\widetilde{A}_{3}=(0,2,4,6)\end{array}$ & Ky Phuc et al. [38], Asady [46] & $\begin{array}{l}\text { Computation complexity } \\
\text { Indecisive ranking for }\left(\widetilde{A}_{1}, \widetilde{A}_{3}\right)\end{array}$ \\
\hline Section 4.2 & Example 20 & $\begin{array}{c}\widetilde{A}_{1}=(-12,1,2) \\
\widetilde{A}_{2}=(-23 / 12,1 / 12,13 / 12) \\
\widetilde{A}_{3}=(-6,0,1,1)\end{array}$ & $\begin{array}{l}\text { Abbasbandy \& Hajjari [74], } \\
\text { Nasseri \& Sohrabi [75] }\end{array}$ & Counterintuition \\
\hline Section 4.3 & Example 22 & $\begin{array}{c}\widetilde{A}_{1}=(1,2,5) \\
\widetilde{A}_{2}=(1,2,2,4)\end{array}$ & $\begin{array}{c}\text { Ky Phuc et al. [38], Asady [46], } \\
\text { Zhang et al. [73] }\end{array}$ & More elaborate in computation \\
\hline
\end{tabular}

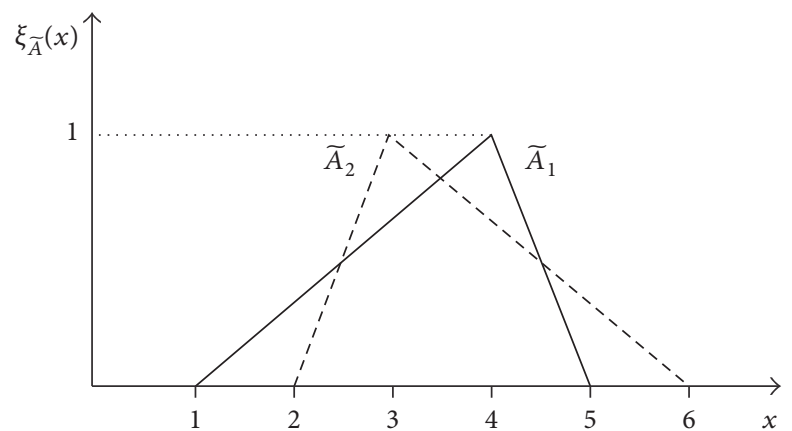

Figure 3: Fuzzy numbers $\widetilde{A}_{1}$ and $\widetilde{A}_{2}$ in Example 15.

but overlapping after flipping and sliding movement. Here, the proposed unified index is contrasted with the most recent work published by $\mathrm{Yu}$ and Dat [48] in 2014 as regards computation simpleness.

According to the unified index in (5), we simply have the results shown in Table $2, \widetilde{A}_{1} \prec \widetilde{A}_{2}\left(\widetilde{A}_{1}^{\prime}>\widetilde{A}_{2}^{\prime}\right)$ at any arbitrary level-of-optimism attitude of data revelation from the decision-maker, $\lambda \in[0,1]$. Yu and Dat [48] advocated the identical ranking result in this case; however, their computation of median values before ranking these two fuzzy numbers is procedure-laborious in practice as reported by some predecessors [58-62].

By the same token, when comparing two normal triangular fuzzy numbers $\widetilde{B}_{1}=(0.1,0.6,0.8)$ and $\widetilde{B}_{2}=(0.2,0.5,0.9)$, taken from [76] and based on the proposed approach, we always have $\widetilde{B}_{1} \prec \widetilde{B}_{2}$, which is coherent with that in $[57,63$,
TABLE 2: Ranking results for Example 15.

\begin{tabular}{lccc}
\hline$\lambda$ & $\mathrm{UI}_{1}^{\lambda}$ & $\mathrm{UI}_{2}^{\lambda}$ & Ranking result \\
\hline 0.0 & 8.333 & 9.167 & $\widetilde{A}_{1} \prec \widetilde{A}_{2}$ and $\widetilde{A}_{1}>\widetilde{A}_{2}^{\prime}$ \\
0.1 & 9.000 & 9.900 & $\widetilde{A}_{1} \prec \widetilde{A}_{2}$ and $\widetilde{A}_{1}^{\prime}>\widetilde{A}_{2}^{\prime}$ \\
0.2 & 9.667 & 10.633 & $\widetilde{A}_{1}<\widetilde{A}_{2}$ and $\widetilde{A}_{1}^{\prime}>\widetilde{A}_{2}^{\prime}$ \\
0.3 & 10.333 & 11.367 & $\widetilde{A}_{1}<\widetilde{A}_{2}$ and $\widetilde{A}_{1}^{\prime}>\widetilde{A}_{2}^{\prime}$ \\
0.4 & 11.000 & 12.100 & $\widetilde{A}_{1}<\widetilde{A}_{2}$ and $\widetilde{A}_{1}>\widetilde{A}_{2}^{\prime}$ \\
0.5 & 11.667 & 12.833 & $\widetilde{A}_{1}<\widetilde{A}_{2}$ and $\widetilde{A}_{1}^{\prime}>\widetilde{A}_{2}^{\prime}$ \\
0.6 & 12.333 & 13.567 & $\widetilde{A}_{1}<\widetilde{A}_{2}$ and $\widetilde{A}_{1}^{\prime}>\widetilde{A}_{2}^{\prime}$ \\
0.7 & 13.000 & 14.300 & $\widetilde{A}_{1}<\widetilde{A}_{2}$ and $\widetilde{A}_{1}^{\prime}>\widetilde{A}_{2}^{\prime}$ \\
0.8 & 13.667 & 15.033 & $\widetilde{A}_{1}<\widetilde{A}_{2}$ and $\widetilde{A}_{1}^{\prime}>\widetilde{A}_{2}$ \\
0.9 & 14.333 & 15.767 & $\widetilde{A}_{1}<\widetilde{A}_{2}$ and $\widetilde{A}_{1}^{\prime}>\widetilde{A}_{2}^{\prime}$ \\
1.0 & 15.000 & 16.500 & $\widetilde{A}_{1}<\widetilde{A}_{2}$ and $\widetilde{A}_{1}^{\prime}>\widetilde{A}_{2}^{\prime}$ \\
\hline
\end{tabular}

77-80]. However, the approaches by R. Chutia and B. Chutia [81] and Deng [82] lead to a counterintuitive result $\widetilde{B}_{2} \prec \widetilde{B}_{1}$.

Example 16. Consider three triangle fuzzy numbers, $\widetilde{A}_{1}=$ $(5,6,7), \widetilde{A}_{2}=(5.9,6,7)$, and $\widetilde{A}_{3}=(6,6,7)$ [39], which are similar and covered with the same right-hand side as displayed in Figure 4. By human instinct, they are easily being discriminated; that is, for the fuzzy numbers and their images, the intuitive and consistent rankings are $\widetilde{A}_{1} \prec$ $\widetilde{A}_{2} \prec \widetilde{A}_{3}$ and $\widetilde{A}_{1}^{\prime}>\widetilde{A}_{2}^{\prime}>\widetilde{A}_{3}^{\prime}$. Therefore, this example is capable of judging the indices' performance if intuition- and consistency-satisfied.

We first check the unified index. Based on (5), Propositions 11 and 12, and Remark 13, the ranking results, listed 
TABLE 3: Ranking results for Example 16.

\begin{tabular}{|c|c|c|c|c|}
\hline$\lambda$ & $\mathrm{UI}_{1}^{\lambda}$ & $\mathrm{UI}_{2}^{\lambda}$ & $\mathrm{UI}_{3}^{\lambda}$ & Ranking result \\
\hline 0.0 & 33.000 & 37.485 & 38.000 & $\widetilde{A}_{1} \prec \widetilde{A}_{2} \prec \widetilde{A}_{3}$ and $\widetilde{A}_{1}>\widetilde{A}_{2}>\widetilde{A}_{3}$ \\
\hline 0.1 & 33.600 & 37.831 & 38.317 & $\widetilde{A}_{1} \prec \widetilde{A}_{2} \prec \widetilde{A}_{3}$ and $\widetilde{A}_{1}^{\prime}>\widetilde{A}_{2}^{\prime}>\widetilde{A}_{3}^{\prime}$ \\
\hline 0.2 & 34.200 & 38.178 & 38.633 & $\widetilde{A}_{1} \prec \widetilde{A}_{2} \prec \widetilde{A}_{3}$ and $\widetilde{A}_{1}^{\prime}>\widetilde{A}_{2}^{\prime}>\widetilde{A}_{3}^{\prime}$ \\
\hline 0.3 & 34.800 & 38.524 & 38.950 & $\widetilde{A}_{1} \prec \widetilde{A}_{2} \prec \widetilde{A}_{3}$ and $\widetilde{A}_{1}^{\prime}>\widetilde{A}_{2}^{\prime}>\widetilde{A}_{3}^{\prime}$ \\
\hline 0.4 & 35.400 & 38.871 & 39.267 & $\widetilde{A_{1}} \prec \widetilde{A_{2}} \prec \widetilde{A}_{3}$ and $\widetilde{A}_{1}>\widetilde{A}_{2}>\widetilde{A}_{3}$ \\
\hline 0.5 & 36.000 & 39.217 & 39.583 & $\widetilde{A}_{1} \prec \widetilde{A}_{2} \prec \widetilde{A}_{3}$ and $\widetilde{A}_{1}^{\prime}>\widetilde{A}_{2}^{\prime}>\widetilde{A}_{3}^{\prime}$ \\
\hline 0.6 & 36.600 & 39.564 & 39.900 & $\widetilde{A_{1}} \prec \widetilde{A}_{2} \prec \widetilde{A}_{3}$ and $\widetilde{A}_{1}>\widetilde{A}_{2}>\widetilde{A}_{3}$ \\
\hline 0.7 & 37.200 & 39.910 & 40.217 & $\widetilde{A_{1}} \prec \widetilde{A_{2}} \prec \widetilde{A_{3}}$ and $\widetilde{A_{1}}>\widetilde{A_{2}}>\widetilde{A}_{3}$ \\
\hline 0.8 & 37.800 & 40.257 & 40.533 & $\widetilde{A}_{1} \prec \widetilde{A}_{2} \prec \widetilde{A}_{3}$ and $\widetilde{A}_{1}>\widetilde{A}_{2}>\widetilde{A}_{3}$ \\
\hline 0.9 & 38.400 & 40.603 & 40.850 & $\widetilde{A}_{1} \prec \widetilde{A}_{2} \prec \widetilde{A}_{3}$ and $\widetilde{A}_{1}^{\prime}>\widetilde{A}_{2}^{\prime}>\widetilde{A}_{3}^{\prime}$ \\
\hline 1.0 & 39.000 & 40.950 & 41.167 & $\widetilde{A_{1}} \prec \widetilde{A_{2}} \prec \widetilde{A}_{3}$ and $\widetilde{A}_{1}^{\prime}>\widetilde{A}_{2}>\widetilde{A}_{3}$ \\
\hline
\end{tabular}

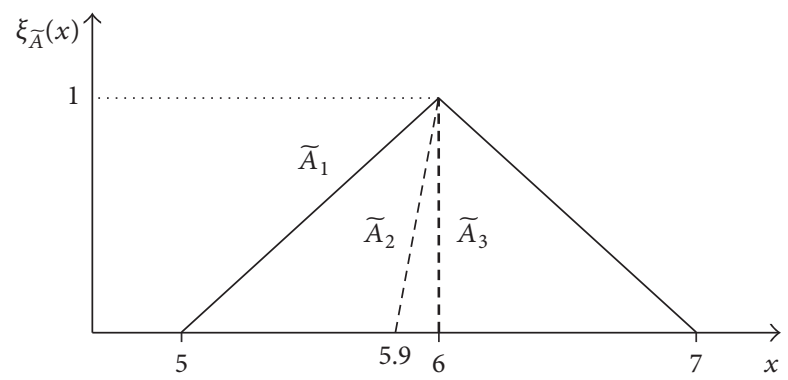

Figure 4: Fuzzy numbers $\widetilde{A}_{1}, \widetilde{A}_{2}$, and $\widetilde{A}_{3}$ in Example 16.

in Table 3 for the fuzzy numbers and their images, affirm the intuitive and consistent outcomes, $\widetilde{A}_{1} \prec \widetilde{A}_{2} \prec \widetilde{A}_{3}$ and $\widetilde{A}_{1}>\widetilde{A}_{2}^{\prime}>\widetilde{A}_{3}^{\prime}$.

In the literature, while many support the intuitive results for ranking the fuzzy numbers $[39,46,57,66,83,84]$, Chen [85] and Chu and Tsao [63] provide a different consequence as $\widetilde{A}_{1} \prec \widetilde{A}_{3} \prec \widetilde{A}_{2}$ and Cheng [57] gives $\widetilde{A}_{3} \prec \widetilde{A}_{2} \prec \widetilde{A}_{1}$, so their counterintuitions are apparent.

Moreover, due to scarcity of methods in the literature for consistently ranking their images, a recent work from Yu and Dat [48] claimed to bridge the gap. Unfortunately, when $\lambda=$ 1, their approach leads to a disparate ranking, $\widetilde{A}_{1} \simeq \widetilde{A}_{2} \simeq$ $\widetilde{A}_{3}\left(\widetilde{A}_{1}^{\prime} \simeq \widetilde{A}_{2}^{\prime} \simeq \widetilde{A}_{3}^{\prime}\right)$, indicating that their index as a whole somewhat lacks reliability.

Example 17. Again, examine three fuzzy numbers, $\widetilde{A}_{1}=$ $(1,3,5), \widetilde{A}_{2}=(2,3,4)$, and $\widetilde{A}_{3}=(1,4,6)$, as shown in Figure 5. Visibly, $\widetilde{A}_{3}=(1,4,6)$ is right way out $\widetilde{A}_{1}$ and $\widetilde{A}_{2}$, so there is no dispute that a capable index should rate $\widetilde{A}_{3}\left(\widetilde{A}_{3}\right)$ as the largest (smallest). The challenging one is to distinguish $\widetilde{A}_{1}$ and $\widetilde{A}_{2}\left(\widetilde{A}_{1}^{\prime}\right.$ and $\left.\widetilde{A}_{2}^{\prime}\right)$ due to their symmetry with respect to $x=3$, identical centroid value, and their geometric enlargement relationship. Actually, majority of the existing ranking measures in category one (evaluating the fuzzy number itself) $\operatorname{rank} \widetilde{A}_{1} \simeq \widetilde{A}_{2}$, and their image ranking is not available. Therefore, this example is to compare the proposed unified index with the category two ranking measures (not only evaluating the fuzzy number itself, but

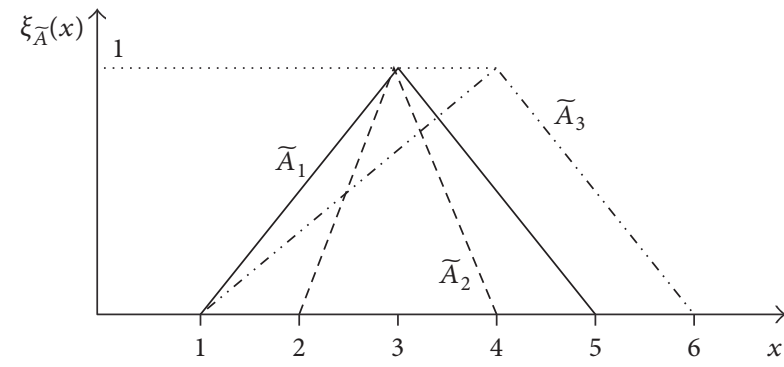

Figure 5: Fuzzy numbers $\widetilde{A}_{1}, \widetilde{A}_{2}$, and $\widetilde{A}_{3}$ in Example 17.

also gauging decision-maker's attitude in regard to specific purposes such as confidence and risk), initiated by Wang and Luo [39], Yu and Dat [48], Yu et al. [65], and Liou and Wang [67], in terms of ranking indices' reliability and consistency.

First, we check the unified index's results in Table 4. Regardless of $\lambda \in[0,1], \widetilde{A}_{3}\left(\widetilde{A}_{3}^{\prime}\right)$ is always the largest (smallest), which confirms human intuition. For the ranking of $\widetilde{A}_{1}$ and $\widetilde{A}_{2}$, dividing from $\lambda=0.5, \widetilde{A}_{1} \simeq \widetilde{A}_{2}\left(\widetilde{A}_{1}^{\prime} \simeq \widetilde{A}_{2}\right)$; the upper part $\lambda \in[0,0.5), \widetilde{A}_{1} \prec \widetilde{A}_{2}\left(\widetilde{A}_{1}^{\prime}>\widetilde{A}_{2}^{\prime}\right)$; the lower part $\lambda \in(0.5,1], \widetilde{A}_{1}>\widetilde{A}_{2}\left(\widetilde{A}_{1}^{\prime} \prec \widetilde{A}_{2}^{\prime}\right)$. Although this result has been proved in Theorem 14, there are still some insightful conclusions to be addressed.

First, this finding is consistent with that of Wang and Luo [39] and Yu et al. [65]. In fact, with respect to the unified index, these results are reasonable because the chosen $\lambda$ value manifests the decision-maker's optimism towards revelation of left- and right-area data. $\lambda \in(0.5,1]$ implies that the right-area data is more preferred by the decision-maker; $\lambda \epsilon$ $[0,0.5)$ represents the notion that the decision-maker is more optimistic regarding the left-area data; $\lambda=0.5$ indicates that the decision-maker is neutral towards preference of data location.

Then, we evaluate the indices proposed by $\mathrm{Yu}$ and Dat [48] and Liou and Wang [67]. While Yu and Dat's work confirms most of the results in Table 4, it does exhibit an apparent counterintuition issue at $\lambda=0$, where it suggests that $\widetilde{A}_{3}$ does not dominate $\widetilde{A}_{2}$; that is, $\widetilde{A}_{2} \simeq \widetilde{A}_{3}\left(\widetilde{A}_{2}^{\prime} \simeq\right.$ $\widetilde{A}_{3}^{\prime}$ ). Moreover, Liou and Wang's index [67] not only afflicts the same shortage of $\mathrm{Yu}$ and Dat's index, but also has 
TABLE 4: Ranking results at different optimism levels in Example 17.

\begin{tabular}{|c|c|c|c|c|}
\hline$\lambda$ & $\mathrm{UI}_{1}^{\lambda}$ & $\mathrm{UI}_{2}^{\lambda}$ & $\mathrm{UI}_{3}^{\lambda}$ & Ranking result \\
\hline 0.0 & 6.000 & 7.500 & 9.167 & $\widetilde{A}_{1} \prec \widetilde{A}_{2} \prec \widetilde{A}_{3}$ and $\widetilde{A}_{1}>\widetilde{A}_{2}>\widetilde{A}_{3}$ \\
\hline 0.1 & 6.600 & 7.800 & 10.083 & $\widetilde{A}_{1} \prec \widetilde{A}_{2} \prec \widetilde{A}_{3}$ and $\widetilde{A}_{1}^{\prime}>{\widetilde{A_{2}}}_{2}^{\prime}>\widetilde{A}_{3}^{\prime}$ \\
\hline 0.2 & 7.200 & 8.100 & 11.000 & $\widetilde{A}_{1} \prec \widetilde{A}_{2} \prec \widetilde{A}_{3}$ and $\widetilde{A}_{1}^{\prime}>\widetilde{A}_{2}^{\prime}>\widetilde{A}_{3}^{\prime}$ \\
\hline 0.3 & 7.800 & 8.400 & 11.917 & $\widetilde{A}_{1} \prec \widetilde{A}_{2} \prec \widetilde{A}_{3}$ and $\widetilde{A}_{1}^{\prime}>\widetilde{A}_{2}^{\prime}>\widetilde{A}_{3}^{\prime}$ \\
\hline 0.4 & 8.400 & 8.700 & 12.833 & $\widetilde{A}_{1} \prec \widetilde{A_{2}} \prec \widetilde{A}_{3}$ and $\widetilde{A}_{1}>\widetilde{A}_{2}>\widetilde{A}_{3}$ \\
\hline 0.5 & 9.000 & 9.000 & 13.750 & $\widetilde{\mathbf{A}}_{1} \simeq \widetilde{\mathbf{A}}_{2} \prec \widetilde{\mathbf{A}}_{3}$ and $\widetilde{\mathbf{A}}_{1}^{\prime} \simeq \widetilde{\mathbf{A}}_{2}^{\prime}>\widetilde{\mathbf{A}}_{3}^{\prime}$ \\
\hline 0.6 & 9.600 & 9.300 & 14.667 & $\widetilde{A_{2}} \prec \widetilde{A_{1}} \prec \widetilde{A_{3}}$ and $\widetilde{A}_{1}>\widetilde{A}_{2}>\widetilde{A}_{3}$ \\
\hline 0.7 & 10.200 & 9.600 & 15.583 & $\widetilde{A}_{2} \prec \widetilde{A}_{1} \prec \widetilde{A}_{3}$ and $\widetilde{A}_{1}^{\prime}>\widetilde{A}_{2}>\widetilde{A}_{3}$ \\
\hline 0.8 & 10.800 & 9.900 & 16.500 & $\widetilde{A}_{2} \prec \widetilde{A}_{1} \prec \widetilde{A}_{3}$ and $\widetilde{A}_{1}^{\prime}>\widetilde{A}_{2}^{\prime}>\widetilde{A}_{3}^{\prime}$ \\
\hline 0.9 & 11.400 & 10.200 & 17.417 & $\widetilde{A}_{2} \prec \widetilde{A}_{1} \prec \widetilde{A}_{3}$ and $\widetilde{A}_{1}^{\prime}>\widetilde{A}_{2}^{\prime}>\widetilde{A}_{3}^{\prime}$ \\
\hline 1.0 & 12.000 & 10.500 & 18.333 & $\widetilde{A_{2}} \prec \widetilde{A_{1}} \prec \widetilde{A_{3}}$ and $\widetilde{A}_{1}^{\prime}>\widetilde{A}_{2}>\widetilde{A}_{3}$ \\
\hline
\end{tabular}

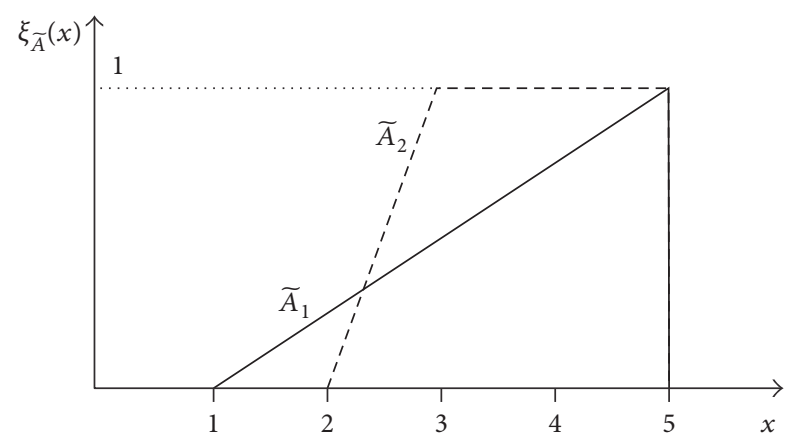

Figure 6: Fuzzy numbers $\widetilde{A}_{1}$ and $\widetilde{A}_{2}$ in Example 18.

shown inconsistent results for ranking the fuzzy numbers and their images due to the index's limited definition and generalization.

4.2. Ranking for Normal Triangular-and-Trapezoid Mixed Fuzzy Numbers. Here, the proposed unified index is used to broaden the ranking comparisons to normal triangular-andtrapezoid mixed fuzzy numbers. The cases from the literature that have one trapezoid mixed with one triangular fuzzy number, followed by two examples with two triangular fuzzy numbers, are investigated.

Example 18. Compare a triangular fuzzy number $\widetilde{A}_{1}=$ $(1,5,5)$ overlapping with a trapezoidal fuzzy number $\widetilde{A}_{2}=$ $(2,3,5,5)$, as shown in Figure 6 . Of ten existing measures that have been studied in this case, three (30\%) support $\widetilde{A}_{1} \prec \widetilde{A}_{2}$ $[30,66,86]$ and seven $(70 \%)$ stand for $\widetilde{A}_{1}>\widetilde{A}_{2}[47,53,63,73$, $74,83,87]$. Clearly, this stark contrast outcome is intriguing for further investigation. Therefore, in this example, we first attempt to explain the predecessors' conflicting consequence by using the unified index. Then, the index itself will be compared with the recent work proposed by Zhang et al. in 2014 [73] to lay out their result similarity as well as their performance with regard to computation easiness and image consistency.

Table 5 is the ranking results of using the unified index, where $\lambda \in[0,0.8], \widetilde{A}_{1}>\widetilde{A}_{2}$ and $\lambda \in[0.9,1], \widetilde{A}_{1} \prec \widetilde{A}_{2}$. Once
TABLE 5: Ranking results at different optimism levels in Example 18.

\begin{tabular}{lccc}
\hline$\lambda$ & $\mathrm{UI}_{1}^{\lambda}$ & $\mathrm{UI}_{2}^{\lambda}$ & Ranking result \\
\hline 0.0 & 11.000 & 9.333 & $\widetilde{A}_{1}>\widetilde{A}_{2}$ \\
0.1 & 11.733 & 10.267 & $\widetilde{A}_{1}>\widetilde{A}_{2}$ \\
0.2 & 12.467 & 11.200 & $\widetilde{A}_{1}>\widetilde{A}_{2}$ \\
0.3 & 13.200 & 12.133 & $\widetilde{A}_{1}>\widetilde{A}_{2}$ \\
0.4 & 13.933 & 13.067 & $\widetilde{A}_{1}>\widetilde{A}_{2}$ \\
0.5 & 14.667 & 14.000 & $\widetilde{A}_{1}>\widetilde{A}_{2}$ \\
0.6 & 15.400 & 14.933 & $\widetilde{A}_{1}>\widetilde{A}_{2}$ \\
0.7 & 16.133 & 15.867 & $\widetilde{A}_{1}>\widetilde{A}_{2}$ \\
0.8 & 16.867 & 16.800 & $\widetilde{A}_{1}>\widetilde{A}_{2}$ \\
0.9 & 17.600 & 17.733 & $\widetilde{A}_{1} \prec \widetilde{A}_{2}$ \\
1.0 & 18.333 & 18.667 & $\widetilde{A}_{1} \prec \widetilde{A}_{2}$ \\
\hline
\end{tabular}

more, the chosen $\lambda$ value manifests the decision-maker's optimism towards revelation of the left-and-right area of fuzzy data. From the $\lambda$-probability point of view, around $80 \%$ support $\widetilde{A}_{1}>\widetilde{A}_{2}$ and $20 \%$ favor $\widetilde{A}_{1} \prec \widetilde{A}_{2}$. In fact, this result, providing a level-of-optimism attitude-based explanation for conflicts among the comparison, is interesting to be approximate with aforementioned percentages obtained from the literature conclusions. Moreover, it is also similar to Zhang et al.s [73] result who uses a preference-probability relation to explain the uncertainty level of the comparison; with seven intricate and somewhat complicated steps, they concluded $\widetilde{A_{1}}>\widetilde{A}_{2}$ with a confidence degree of $73 \%$ and $\widetilde{A_{1}} \prec \widetilde{A_{2}}$ with $27 \%$.

Finally, it is worth mentioning that as opposed to the unified index, Zhang et al.s [73] seven-step algorithm for ranking fuzzy numbers not only suffers a computationcomplexity problem, but also lacks capacity for ranking the fuzzy-number image.

Example 19. Taken from [38] and shown in Figure 7, one trapezoid fuzzy number, $\widetilde{A}_{3}=(0,2,4,6)$, mingled with two triangular fuzzy numbers, $\widetilde{A}_{1}=(0,3,6)$ and $\widetilde{A}_{2}=(-1,0,2)$, is considered in this example. Noticeably, $\widetilde{A}_{2}$ left distances away from $\widetilde{A}_{1}$ and $\widetilde{A}_{3}$, so there is no argument that a reliable 
TABLE 6: Ranking results of the three fuzzy numbers in Example 19.

\begin{tabular}{lcccc}
\hline$\lambda$ & $\mathrm{UI}_{1}^{\lambda}$ & $\mathrm{UI}_{2}^{\lambda}$ & $\mathrm{UI}_{3}^{\lambda}$ & Ranking result \\
\hline 0.0 & 4.500 & 0.167 & 3.000 & $\widetilde{A}_{1}>\widetilde{A}_{3}>\widetilde{A}_{2}$ and $\widetilde{A}_{1}^{\prime}<\widetilde{A}_{3}<\widetilde{A}_{2}^{\prime}$ \\
0.1 & 0.183 & 4.200 & $\widetilde{A}_{1}>\widetilde{A}_{3}>\widetilde{A}_{2}$ and $\widetilde{A}_{1}^{\prime}<\widetilde{A}_{3}^{\prime}<\widetilde{A}_{2}^{\prime}$ \\
0.2 & 0.200 & 0.400 & $\widetilde{A}_{1}>\widetilde{A}_{3}>\widetilde{A}_{2}$ and $\widetilde{A}_{1}^{\prime}<\widetilde{A}_{3}^{\prime}<\widetilde{A}_{2}^{\prime}$ \\
0.3 & 6.300 & 0.217 & $\widetilde{A}_{1}>\widetilde{A}_{3}>\widetilde{A}_{2}$ and $\widetilde{A}_{1}^{\prime}<\widetilde{A}_{3}^{\prime}<\widetilde{A}_{2}^{\prime}$ \\
0.4 & 7.200 & 0.233 & 7.800 & $\widetilde{A}_{1}>\widetilde{A}_{3}>\widetilde{A}_{2}$ and $\widetilde{A}_{1}^{\prime}<\widetilde{A}_{3}^{\prime}<\widetilde{A}_{2}^{\prime}$ \\
$\mathbf{0 . 5}$ & 8.100 & $\mathbf{0 . 2 5 0}$ & $\mathbf{9 . 0 0 0}$ & $\widetilde{\mathbf{A}}_{1} \simeq \widetilde{\mathbf{A}}_{3}>\widetilde{\mathbf{A}}_{2}$ and $\widetilde{\mathbf{A}}_{1}^{\prime} \simeq \widetilde{\mathbf{A}}_{3}^{\prime}<\widetilde{\mathrm{A}}_{2}^{\prime}$ \\
0.6 & $\mathbf{9 . 0 0 0}$ & 0.267 & 10.200 & $\widetilde{A}_{3}>\widetilde{A}_{1}>\widetilde{A}_{2}$ and $\widetilde{A}_{3}^{\prime}<\widetilde{A}_{1}^{\prime}<\widetilde{A}_{2}^{\prime}$ \\
0.7 & 9.900 & 0.283 & 11.400 & $\widetilde{A}_{3}>\widetilde{A}_{1}>\widetilde{A}_{2}$ and $\widetilde{A}_{3}^{\prime}<\widetilde{A}_{1}<\widetilde{A}_{2}^{\prime}$ \\
0.8 & 10.800 & 0.300 & 12.600 & $\widetilde{A}_{3}>\widetilde{A}_{1}>\widetilde{A}_{2}$ and $\widetilde{A}_{3}^{\prime}<\widetilde{A}_{1}<\widetilde{A}_{2}^{\prime}$ \\
0.9 & 11.700 & 0.317 & 13.800 & $\widetilde{A}_{3}>\widetilde{A}_{1}>\widetilde{A}_{2}$ and $\widetilde{A}_{3}^{\prime}<\widetilde{A}_{1}^{\prime}<\widetilde{A}_{2}^{\prime}$ \\
1.0 & 12.600 & 0.333 & $\widetilde{A}_{3}>\widetilde{A}_{1}>\widetilde{A}_{2}$ and $\widetilde{A}_{3}^{\prime}<\widetilde{A}_{1}^{\prime}<\widetilde{A}_{2}^{\prime}$ \\
\hline
\end{tabular}

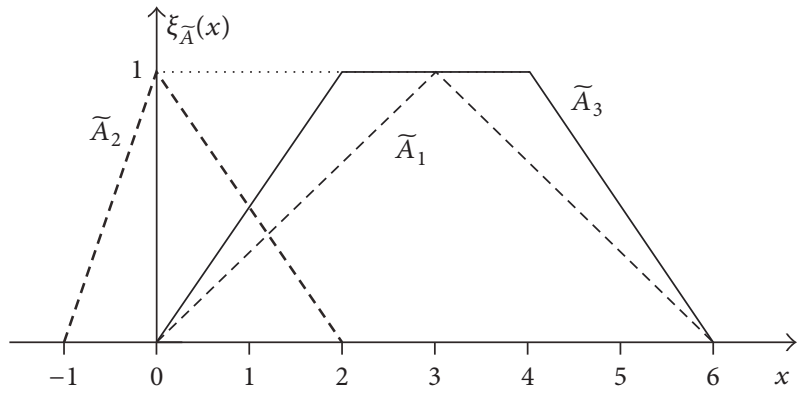

Figure 7: Fuzzy numbers $\widetilde{A}_{1}, \widetilde{A}_{2}$, and $\widetilde{A}_{3}$ in Example 19.

index should discriminate $\widetilde{A}_{2}\left(\widetilde{A}_{2}^{\prime}\right)$ as the smallest (largest). The question is the rating result of the triangular fuzzy number $\widetilde{A}_{1}$ and the trapezoid fuzzy number $\widetilde{A}_{3}$ and their images. Therefore, this example is to compare the unified index with the recent works of Asady in 2010 and Ky Phuc et al. [38] in 2012 who proposed a deviation-degree ranking measure.

First, we check the unified index's results in Table 6. Regardless of $\lambda \in[0,1], \widetilde{A}_{2}\left(\widetilde{A}_{2}^{\prime}\right)$ is always the smallest (largest), which confirms human intuition. For the ranking of $\widetilde{A}_{1}$ and $\widetilde{A}_{3}$, dividing from $\lambda=0.5, \widetilde{A}_{1} \simeq \widetilde{A}_{3}\left(\widetilde{A}_{1}^{\prime} \simeq \widetilde{A}_{3}^{\prime}\right)$; the upper part $\lambda \in[0,0.5), \widetilde{A}_{1}>\widetilde{A}_{3}\left(\widetilde{A}_{1}^{\prime} \prec \widetilde{A}_{3}^{\prime}\right)$; the lower part $\lambda \in(0.5,1], \widetilde{A}_{1} \prec \widetilde{A}_{3}\left(\widetilde{A}_{1}^{\prime}>\widetilde{A}_{3}^{\prime}\right)$. Literally, this finding (refer to Theorem 14) is consistent with two fuzzy numbers with the same attitude and symmetry, shown in Example 17.

Then, we evaluate Ky Phuc et al.s [38] and Asady's [46] deviation-degree index. Despite the exhausted computation, its capability can only provide the partial result, " $\widetilde{A}_{1}>\widetilde{A}_{2}$ " and " $\widetilde{A}_{3}>\widetilde{A}_{2}$," leaving undecided ranking for $\widetilde{A}_{1}$ and $\widetilde{A}_{3}$. Actually, as mentioned in Section 1, the deviation-degree index, belonging to the category one ranking measure, has the limitation for ranking the fuzzy numbers akin to $\widetilde{A}_{1}$ and $\widetilde{A}_{3}$ that are overlapping and each has axis-of-symmetry property.

Example 20. Additionally, let us consider one trapezoidal fuzzy number, $\widetilde{A}_{3}=(-6,0,1,1)$, blended with two triangular

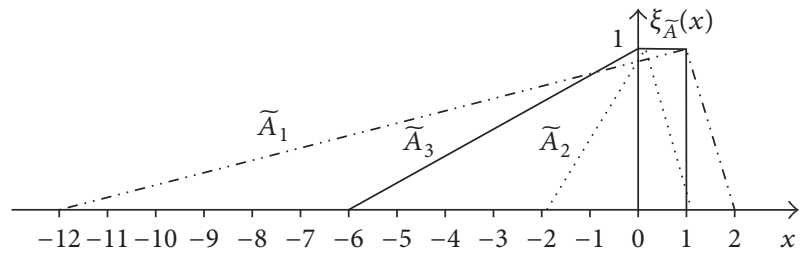

Figure 8: Fuzzy numbers $\widetilde{A}_{1}, \widetilde{A}_{2}$, and $\widetilde{A}_{3}$ in Example 20.

fuzzy numbers, $\widetilde{A}_{1}=(-12,1,2)$ and $\widetilde{A}_{2}=(-23 / 12,1 / 12$, 13/12), which are adapted from [66] and shown in Figure 8. Unlike the previous challenging one that is with a symmetric and triangle-embedded trapezoid shape, they are all leftskewed fuzzy numbers and easy to be distinguished by human perception; that is, $\widetilde{A}_{1} \prec \widetilde{A}_{3} \prec \widetilde{A}_{2}$. Hence, for this subsection, this example is capable of judging the indices' performance if intuition-satisfied.

The result in Table 7, obtained with the unified index, clearly shows that $\widetilde{A}_{1} \prec \widetilde{A}_{3} \prec \widetilde{A}_{2}$, which is identical to previous works in $[46,47,63,64,66,87]$. However, counter results are claimed by Abbasbandy and Hajjari [74] who ranked them as $\widetilde{A}_{1} \simeq \widetilde{A}_{2} \simeq \widetilde{A}_{3}$ and Nasseri and Sohrabi [75] who suggested $\widetilde{A}_{2} \prec \widetilde{A}_{3} \prec \widetilde{A}_{1}$. Both works' counterintuition is obvious.

Example 21. Now, two special cases taken from R. Chutia and B. Chutia [81] are considered. The first set includes $\widetilde{A}_{1}=$ $(0.1,0.1,0.1,0.1 ; 0.8)$ and $\widetilde{A}_{2}=(-0.1,-0.1,-0.1,-0.1 ; 1.0)$ which were ranked as $\widetilde{A}_{1}>\widetilde{A}_{2}$; and the second one includes $\widetilde{B}_{1}=(1,1,1,1 ; 0.5)$ and $\widetilde{B}_{2}=(1,1,1,1 ; 1.0)$ which were ranked as $\widetilde{B}_{1}<\widetilde{B}_{2}$. The proposed unified index also leads to similar conclusions as in $[37,78,81,88-90]$, indicating that the index can effectively work with crisp numbers as well.

4.3. Ranking for Nonlinear Fuzzy Numbers. Finally, although empirical phenomenon and human perception are rather unlikely to gather the nonlinear fuzzy numbers, this more general type can be justifiable for investigating the index's computation easiness as well as adaptability. 
TABLE 7: Ranking results of the three fuzzy numbers in Example 20.

\begin{tabular}{lcccc}
\hline$\lambda$ & $\mathrm{UI}_{1}^{\lambda}$ & $\mathrm{UI}_{2}^{\lambda}$ & $\mathrm{UI}_{3}^{\lambda}$ & Ranking result \\
\hline 0.0 & -18.333 & -0.229 & -4.125 & $\widetilde{A}_{1} \prec \widetilde{A}_{3} \prec \widetilde{A}_{2}$ \\
0.1 & -16.833 & -0.221 & -3.850 & $\widetilde{A}_{1} \prec \widetilde{A}_{3} \prec \widetilde{A}_{2}$ \\
0.2 & -15.333 & -0.213 & -3.575 & $\widetilde{A}_{1} \prec \widetilde{A}_{3} \prec \widetilde{A}_{2}$ \\
0.3 & -13.833 & -0.204 & -3.300 & $\widetilde{A}_{1} \prec \widetilde{A}_{3} \prec \widetilde{A}_{2}$ \\
0.4 & -12.333 & -0.196 & -3.025 & $\widetilde{A}_{1} \prec \widetilde{A}_{3} \prec \widetilde{A}_{2}$ \\
0.5 & -10.833 & -0.188 & -2.750 & $\widetilde{A}_{1} \prec \widetilde{A}_{3} \prec \widetilde{A}_{2}$ \\
0.6 & -9.333 & -0.179 & -2.475 & $\widetilde{A}_{1} \prec \widetilde{A}_{3} \prec \widetilde{A}_{2}$ \\
0.7 & -7.833 & -0.171 & -2.200 & $\widetilde{A}_{1}<\widetilde{A}_{3} \prec \widetilde{A}_{2}$ \\
0.8 & -6.333 & -0.163 & -1.925 & $\widetilde{A}_{1}<\widetilde{A}_{3} \prec \widetilde{A}_{2}$ \\
0.9 & -4.833 & -0.154 & -1.650 & $\widetilde{A}_{1} \prec \widetilde{A}_{3} \prec \widetilde{A}_{2}$ \\
1.0 & -3.333 & -0.146 & -1.375 & $\widetilde{A}_{1}<\widetilde{A}_{3} \prec \widetilde{A}_{2}$ \\
\hline
\end{tabular}

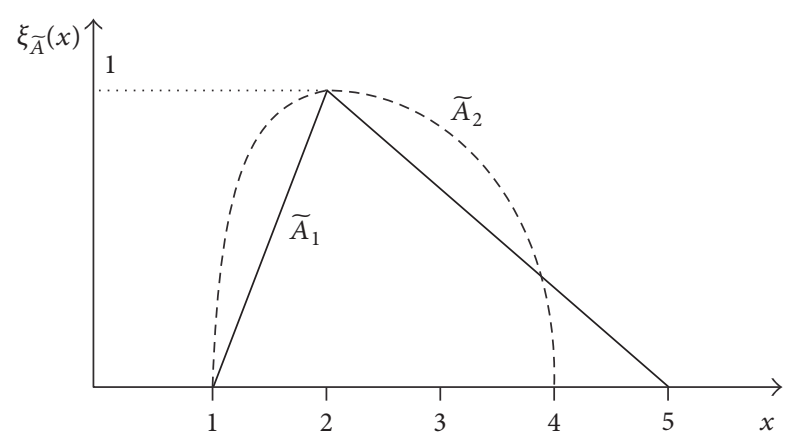

Figure 9: Fuzzy numbers $\widetilde{A}_{1}$ and $\widetilde{A}_{2}$ in Example 22.

Example 22. Let us consider two fuzzy numbers shown in Figure 9, adapted from Liou and Wang [67]: $\widetilde{A}_{1}=(1,2,5)$ and $\widetilde{A}_{2}=(1,2,2,4)$ with a nonlinear membership function

$$
f_{\widetilde{A}_{2}}(x)= \begin{cases}{\left[1-(x-2)^{2}\right]^{1 / 2},} & 1 \leq x \leq 2, \\ {\left[1-\frac{1}{4}(x-2)^{2}\right]^{1 / 2},} & 2 \leq x \leq 4 \\ 0, & \text { otherwise }\end{cases}
$$

In this nonlinear case, by using the unified index, the conclusions in Table 8, $\widetilde{A}_{1}>\widetilde{A}_{2}\left(\widetilde{A}_{1} \prec \widetilde{A}_{2}^{\prime}\right)$ for $\lambda \in[0,1]$, do not add much complexity for the computation. Obviously, previous proposed measures in $[53,63,66,67,76]$ possess the same conclusion and computation easiness. However, in recent works, Ky Phuc et al. [38], Asady [46], and Zhang et al. [73], their indices become more complicated and elaborate for ranking the nonlinear fuzzy numbers as well as their images.

\section{Conclusions}

Numerous indices for fuzzy-data comparisons and rankings have been widely implemented to resolve decisive problems that are evaluated under resources constraint and with to some extent linguistic preference of multiattribute, realized from users' perspectives, as well as subjective quantification of multiple characteristics, assessed from decision-makers. However, counterintuition, computation complexity, less reliability, and/or inconsistency on their fuzzy-number rating
TABLE 8: Ranking results at different optimism levels in Example 22.

\begin{tabular}{lccc}
\hline$\lambda$ & $\mathrm{UI}_{1}^{\lambda}$ & $\mathrm{UI}_{2}^{\lambda}$ & Ranking result \\
\hline 0.0 & 6.750 & 2.945 & $\widetilde{A}_{1}>\widetilde{A}_{2}$ and $\widetilde{A}_{1} \prec \widetilde{A}_{2}$ \\
0.1 & 7.650 & 3.516 & $\widetilde{A}_{1}>\widetilde{A}_{2}$ and $\widetilde{A}_{1}^{\prime} \prec \widetilde{A}_{2}^{\prime}$ \\
0.2 & 8.550 & 4.087 & $\widetilde{A}_{1}>\widetilde{A}_{2}$ and $\widetilde{A}_{1} \prec \widetilde{A}_{2}$ \\
0.3 & 9.450 & 4.658 & $\widetilde{A}_{1}>\widetilde{A}_{2}$ and $\widetilde{A}_{1} \prec \widetilde{A}_{2}$ \\
0.4 & 10.350 & 5.230 & $\widetilde{A}_{1}>\widetilde{A}_{2}$ and $\widetilde{A}_{1} \prec \widetilde{A}_{2}$ \\
0.5 & 11.250 & 5.801 & $\widetilde{A}_{1}>\widetilde{A}_{2}$ and $\widetilde{A}_{1}^{\prime} \prec \widetilde{A}_{2}^{\prime}$ \\
0.6 & 12.150 & 6.372 & $\widetilde{A}_{1}>\widetilde{A}_{2}$ and $\widetilde{A}_{1} \prec \widetilde{A}_{2}^{\prime}$ \\
0.7 & 13.050 & 6.943 & $\widetilde{A}_{1}>\widetilde{A}_{2}$ and $\widetilde{A}_{1} \prec \widetilde{A}_{2}$ \\
0.8 & 13.950 & 7.515 & $\widetilde{A}_{1}>\widetilde{A}_{2}$ and $\widetilde{A}_{1} \prec \widetilde{A}_{2}$ \\
0.9 & 14.850 & 8.086 & $\widetilde{A}_{1}>\widetilde{A}_{2}$ and $\widetilde{A}_{1} \prec \widetilde{A}_{2}$ \\
1.0 & 15.750 & 8.657 & $\widetilde{A}_{1}>\widetilde{A}_{2}$ and $\widetilde{A}_{1} \prec \widetilde{A}_{2}$ \\
\hline
\end{tabular}

outcomes have hampered their comprehensive implementation. To lessen their exhibited ranking weaknesses, this paper develops a unified index that multiplies weighted-mean and weighted-area discriminatory components of a fuzzy number, respectively, called centroid value (a measure that values the fuzzy number itself) and attitude-incorporated left-and-right area (a fuzzy-number measure that also reflects on the decision-maker's attitude as regards data revelation). From theoretical proofs and comparative studies, this unified index has demonstrated four advantages for ranking fuzzy numbers.

First, ranking results of the unified index support the human-intuition judgement. Secondly, it shows computation easiness regardless of different types of fuzzy numbers. It can be noted that this computation simplicity becomes crucial for multiagents-multicriteria decision-making problems, which normally involve numerous comparisons and analyses of fuzzy numbers. Thirdly, the unified index can provide a level-of-optimism attitude-based explanation for ranking conflicts among the literature. Most importantly, the unified index possesses the consistency property for ranking fuzzy numbers and their images as well as for symmetric fuzzy numbers with an identical altitude. Literally, in fields of computer vision and image pattern recognition, this property has been a rather critical one for accurate matching and/or retrieval of information.

\section{Conflicts of Interest}

The author declares that there are no conflicts of interest regarding the publication of this paper.

\section{Acknowledgments}

This work was partially supported by Lac Hong University under Decision no. 918/QD-DHLH.

\section{References}

[1] M.-H. Shu and H.-C. Wu, "Fuzzy $\bar{X}$ and $R$ control charts: fuzzy dominance approach," Computers and Industrial Engineering, vol. 61, no. 3, pp. 676-685, 2011. 
[2] M.-H. Shu, T.-L. Nguyen, and B.-M. Hsu, "Fuzzy MaxGWMA chart for identifying abnormal variations of on-line manufacturing processes with imprecise information," Expert Systems with Applications, vol. 41, no. 4, pp. 1342-1356, 2014.

[3] P. Singh, "A new approach for the ranking of fuzzy sets with different heights," Journal of Applied Research and Technology, vol. 10, pp. 941-949, 2012.

[4] V. F. Yu, H. T. X. Chi, and C.-W. Shen, "Ranking fuzzy numbers based on epsilon-deviation degree," Applied Soft Computing Journal, vol. 13, no. 8, pp. 3621-3627, 2013.

[5] S. A. H. Soliman and A. A. H. Mantawy, Modern Optimization Techniques with Applications in Electric Power Systems, Springer, New York, NY, USA, 2012.

[6] J. J. Buckley and T. Feuring, "Fuzzy differential equations," Fuzzy Sets and Systems, vol. 110, no. 1, pp. 43-54, 2000.

[7] B. Bede and S. G. Gal, "Generalizations of the differentiability of fuzzy-number-valued functions with applications to fuzzy differential equations," Fuzzy Sets and Systems, vol. 151, no. 3, pp. 581-599, 2005.

[8] B. Bede, I. J. Rudas, and A. L. Bencsik, "First order linear fuzzy differential equations under generalized differentiability," Information Sciences, vol. 177, no. 7, pp. 1648-1662, 2007.

[9] M. T. Mizukoshi, L. C. Barros, Y. Chalco-Cano, H. RománFlores, and R. C. Bassanezi, "Fuzzy differential equations and the extension principle," Information Sciences, vol. 177, no. 17, pp. 3627-3635, 2007.

[10] Y. Chalco-Cano and H. Román-Flores, "On new solutions of fuzzy differential equations," Chaos, Solitons and Fractals, vol. 38, no. 1, pp. 112-119, 2008.

[11] N. Gasilov, Ş. E. Amrahov, and A. G. Fatullayev, "Solution of linear differential equations with fuzzy boundary values," Fuzzy Sets and Systems, vol. 257, pp. 169-183, 2014.

[12] D. Qiu, W. Zhang, and C. Lu, "On fuzzy differential equations in the quotient space of fuzzy numbers," Fuzzy Sets and Systems, vol. 295, pp. 72-98, 2016.

[13] M. Mosleh and M. Otadi, "Approximate solution of fuzzy differential equations under generalized differentiability," Applied Mathematical Modelling, vol. 39, no. 10-11, pp. 3003-3015, 2015.

[14] A. Khastan and R. Rodríguez-López, "On periodic solutions to first order linear fuzzy differential equations under differential inclusions' approach," Information Sciences, vol. 322, Article ID 11597, pp. 31-50, 2015.

[15] P. Balasubramaniam and S. Muralisankar, "Existence and uniqueness of fuzzy solution for the nonlinear fuzzy integrodifferential equations," Applied Mathematics Letters, vol. 14, no. 4, pp. 455-462, 2001.

[16] S. Abbasbandy, J. J. Nieto, and M. Alavi, "Tuning of reachable set in one dimensional fuzzy differential inclusions," Chaos, Solitons and Fractals, vol. 26, no. 5, pp. 1337-1341, 2005.

[17] H. R. Rahimi, M. Khezerloo, and S. Khezerloo, "Approximating the fuzzy solution of the non-linear fuzzy Volterra integrodifferential equation using fixed point theorems," International Journal of Industrial Mathematics, vol. 3, pp. 227-236, 2011.

[18] T. Allahviranloo, S. Abbasbandy, O. Sedaghgatfar, and P. Darabi, "A new method for solving fuzzy integro-differential equation under generalized differentiability," Neural Computing and Applications, vol. 21, no. 1, pp. 191-196, 2012.

[19] R. Alikhani, F. Bahrami, and A. Jabbari, "Existence of global solutions to nonlinear fuzzy Volterra integro-differential equations," Nonlinear Analysis: Theory, Methods and Applications, vol. 75, no. 4, pp. 1810-1821, 2012.
[20] B. Babayar-Razlighi and B. Soltanalizadeh, "Numerical solution for system of singular nonlinear Volterra integro-differential equations by Newton-Product method," Applied Mathematics and Computation, vol. 219, no. 15, pp. 8375-8383, 2013.

[21] N. V. Hoa and N. D. Phu, "Fuzzy functional integro-differential equations under generalized H-differentiability," Journal of Intelligent and Fuzzy Systems, vol. 26, no. 4, pp. 2073-2085, 2014.

[22] R. Alikhani and F. Bahrami, "Global solutions of fuzzy integrodifferential equations under generalized differentiability by the method of upper and lower solutions," Information Sciences, vol. 295, pp. 600-608, 2015.

[23] T.-C. Chu and Y.-C. Lin, "A fuzzy TOPSIS method for robot selection," International Journal of Advanced Manufacturing Technology, vol. 21, no. 4, pp. 284-290, 2003.

[24] S.-Y. Chou and Y.-H. Chang, "A decision support system for supplier selection based on a strategy-aligned fuzzy SMART approach," Expert Systems with Applications, vol. 34, no. 4, pp. 2241-2253, 2008.

[25] B. Wang, H. Xiong, and C. Jiang, "A multicriteria decision making approach based on fuzzy theory and credibility mechanism for logistics center location selection," The Scientific World Journal, vol. 2014, Article ID 347619, 2014.

[26] S.-Y. Chou, Y.-H. Chang, and C.-Y. Shen, "A fuzzy simple additive weighting system under group decision-making for facility location selection with objective/subjective attributes," European Journal of Operational Research, vol. 189, no. 1, pp.132$145,2008$.

[27] A. Azadeh, M. Osanloo, and M. Ataei, "A new approach to mining method selection based on modifying the Nicholas technique," Applied Soft Computing Journal, vol. 10, no. 4, pp. 1040-1061, 2010.

[28] S.-H. Sheu, C.-J. Huang, and T.-S. Hsu, "Extended maximum generally weighted moving average control chart for monitoring process mean and variability," Computers and Industrial Engineering, vol. 62, no. 1, pp. 216-225, 2012.

[29] M. Gülbay and C. Kahraman, "An alternative approach to fuzzy control charts: direct fuzzy approach," Information Sciences, vol. 177, no. 6, pp. 1463-1480, 2007.

[30] C.-B. Cheng, "Fuzzy process control: construction of control charts with fuzzy numbers," Fuzzy Sets and Systems, vol. 154, no. 2, pp. 287-303, 2005.

[31] T. L. Nguyen, Fuzzy Control Charts - Construction and Application [Ph.D. thesis], National Kaohsiung University of Applied Sciences, Kaohsiung, Taiwan, 2014.

[32] Z. Wang, Y. Hu, Y. Wang, C. Dong, and Z. Pang, "Cutting force predication based on integration of symmetric fuzzy number and finite element method," The Scientific World Journal, vol. 2014, Article ID 234150, 2014.

[33] M.-L. Tseng, "An assessment of cause and effect decisionmaking model for firm environmental knowledge management capacities in uncertainty," Environmental Monitoring and Assessment, vol. 161, no. 1-4, pp. 549-564, 2010.

[34] M.-L. Tseng, "Using a hybrid MCDM model to evaluate firm environmental knowledge management in uncertainty," Applied Soft Computing Journal, vol. 11, no. 1, pp. 1340-1352, 2011.

[35] M.-L. Tseng, "Green supply chain management with linguistic preferences and incomplete information," Applied Soft Computing Journal, vol. 11, no. 8, pp. 4894-4903, 2011.

[36] M. Dağdeviren, S. Yavuz, and N. Kilinç, "Weapon selection using the AHP and TOPSIS methods under fuzzy environment," Expert Systems with Applications, vol. 36, no. 4, pp. 81438151, 2009. 
[37] R. Abbasi Shureshjani and M. Darehmiraki, "A new parametric method for ranking fuzzy numbers," Indagationes Mathematicae, vol. 24, no. 3, pp. 518-529, 2013.

[38] P. N. Ky Phuc, V. F. Yu, S.-Y. Chou, and L. Q. Dat, "Analyzing the ranking method for L-R fuzzy numbers based on deviation degree," Computers and Industrial Engineering, vol. 63, no. 4, pp. 1220-1226, 2012.

[39] Y.-M. Wang and Y. Luo, "Area ranking of fuzzy numbers based on positive and negative ideal points," Computers \& Mathematics with Applications, vol. 58, no. 9, pp. 1769-1779, 2009.

[40] G. Bortolan and R. Degani, "A review of some methods for ranking fuzzy subsets," Fuzzy Sets and Systems, vol. 15, no. 1, pp. $1-19,1985$.

[41] M. Brunelli and J. Mezei, "How different are ranking methods for fuzzy numbers? A numerical study," International Journal of Approximate Reasoning, vol. 54, no. 5, pp. 627-639, 2013.

[42] N. R. Shankar and P. P. B. Rao, "Ranking fuzzy numbers with a distance method using Circumcenter of Centroids and an index of modality," Advances in Fuzzy Systems, vol. 2011, Article ID 178308, 7 pages, 2011.

[43] X. Wang and E. E. Kerre, "Reasonable properties for the ordering of fuzzy quantities (I)," Fuzzy Sets and Systems, vol. 118, no. 3, pp. 375-385, 2001.

[44] X. Wang and E. E. Kerre, "Reasonable properties for the ordering of fuzzy quantities (II)," Fuzzy Sets and Systems, vol. 118, no. 3, pp. 387-405, 2001.

[45] S. J. Chen and C. L. Hwang, Fuzzy Multiple Attribute Decision Making: Methods and Applications, vol. 375 of Lecture Notes in Economics and Mathematical Systems, Springer, New York, NY, USA, 1992.

[46] B. Asady, "The revised method of ranking LR fuzzy number based on deviation degree," Expert Systems with Applications, vol. 37, no. 7, pp. 5056-5060, 2010.

[47] B. Asady, "Revision of distance minimization method for ranking of fuzzy numbers," Applied Mathematical Modelling, vol. 35, no. 3, pp. 1306-1313, 2011.

[48] V. F. Yu and L. Q. Dat, "An improved ranking method for fuzzy numbers with integral values," Applied Soft Computing Journal, vol. 14, pp. 603-608, 2014.

[49] R. Jain, "A procedure for multi-aspect decision making using fuzzy sets," International Journal of Systems Science, vol. 8, no. 1, pp. 1-7, 1977.

[50] J. D. Kim, E. L. Moon, E. Jeong, and D. H. Hong, "Ranking methods for fuzzy numbers: the solution to Brunelli and Mezei's conjecture," Fuzzy Sets and Systems, vol. 315, pp. 109-113, 2017.

[51] Q. Gu and Z. Xuan, "A new approach for ranking fuzzy numbers based on possibility theory," Journal of Computational and Applied Mathematics, vol. 309, pp. 674-682, 2017.

[52] M. L. Wang, H. F. Wang, and C. L. Lin, "Ranking fuzzy number based on lexicographic screening procedure," International Journal of Information Technology \& Decision Making, vol. 4, no. 4, pp. 663-678, 2005.

[53] A. M. Nejad and M. Mashinchi, "Ranking fuzzy numbers based on the areas on the left and the right sides of fuzzy number," Computers \& Mathematics with Applications, vol. 61, no. 2, pp. 431-442, 2011.

[54] C. B. Chen and C. M. Klein, "An efficient approach to solving fuzzy MADM problems," Fuzzy Sets and Systems, vol. 88, no. 1, pp. 51-67, 1997.

[55] R. R. Yager, "A procedure for ordering fuzzy subsets of the unit interval," Information Sciences, vol. 24, no. 2, pp. 143-161, 1981.
[56] E. S. Lee and R.-J. Li, "Comparison of fuzzy numbers based on the probability measure of fuzzy events," Computers \& Mathematics with Applications, vol. 15, no. 10, pp. 887-896, 1988.

[57] C.-H. Cheng, "A new approach for ranking fuzzy numbers by distance method," Fuzzy Sets and Systems, vol. 95, no. 3, pp. 307317, 1998.

[58] R. Saneifard and R. Saneifard, "The median value of fuzzy numbers and its applications in decision making," Journal of Fuzzy Set Valued Analysis, Article ID jfsva-00051, 9 pages, 2012.

[59] Z. J. Zhang, Z. J. Wang, and B. H. Zhang, "Studies on median value of fuzzy numbers based on confidence level," in Proceedings of the 7th International Conference on Machine Learning and Cybernetics, ICMLC, pp. 588-593, Kunming, China, 12-15 July 2008.

[60] S. Bodjanova, "Median value and median interval of a fuzzy number," Information Sciences, vol. 172, no. 1-2, pp. 73-89, 2005.

[61] S. Bodjanova, "Median alpha-levels of a fuzzy number," Fuzzy Sets and Systems, vol. 157, no. 7, pp. 879-891, 2006.

[62] M. Yamashiro, “The median for a L-R fuzzy number," Microelectronics Reliability, vol. 35, no. 2, pp. 269-271, 1995.

[63] T.-C. Chu and C.-T. Tsao, "Ranking fuzzy numbers with an area between the centroid point and original point," Computers \& Mathematics with Applications, vol. 43, no. 1-2, pp. 111-117, 2002.

[64] Y.-J. Wang and H.-S. Lee, "The revised method of ranking fuzzy numbers with an area between the centroid and original points," Computers and Mathematics with Applications, vol. 55, no. 9, pp. 2033-2042, 2008.

[65] V. F. Yu, H. T. Chi, L. Q. Dat, P. N. Phuc, and C.-W. Shen, "Ranking generalized fuzzy numbers in fuzzy decision making based on the left and right transfer coefficients and areas," Applied Mathematical Modelling, vol. 37, no. 16-17, pp. 8106-8117, 2013.

[66] Z.-X. Wang, Y.-J. Liu, Z.-P. Fan, and B. Feng, "Ranking L-R fuzzy number based on deviation degree," Information Sciences, vol. 179, no. 13, pp. 2070-2077, 2009.

[67] T.-S. Liou and M. J. Wang, "Ranking fuzzy numbers with integral value," Fuzzy Sets and Systems, vol. 50, no. 3, pp. 247255, 1992.

[68] S. Das and D. Guha, "A centroid-based ranking method of trapezoidal intuitionistic fuzzy numbers and its application to MCDM problems," Fuzzy Information and Engineering, vol. 8, no. 1, pp. 41-74, 2016.

[69] H. Zimmermann, Fuzzy Set Theory-and Its Applications, Kluwer Academic Publishers, Boston, Mass, USA, 3rd edition, 2001.

[70] K. H. Lee, First Course on Fuzzy Theory and Applications, Springer, 1st edition, 2005.

[71] H. B. Mitchell and P. A. Schaefer, "On ordering fuzzy numbers," International Journal of Intelligent Systems, vol. 15, no. 11, pp. 981-993, 2000.

[72] P. Fortemps and M. Roubens, "Ranking and defuzzification methods based on area compensation," Fuzzy Sets and Systems, vol. 82, no. 3, pp. 319-330, 1996.

[73] F. Zhang, J. Ignatius, C. P. Lim, and Y. Zhao, "A new method for ranking fuzzy numbers and its application to group decision making," Applied Mathematical Modelling, vol. 38, no. 4, pp. 1563-1582, 2014.

[74] S. Abbasbandy and T. Hajjari, "A new approach for ranking of trapezoidal fuzzy numbers," Computers \& Mathematics with Applications, vol. 57, no. 3, pp. 413-419, 2009. 
[75] S. H. Nasseri and M. Sohrabi, "Ranking fuzzy numbers by using of gration," Australian Journal of Basic and Applied Sciences, vol. 4, no. 4, pp. 658-664, 2010.

[76] R. Ezzati, T. Allahviranloo, S. Khezerloo, and M. Khezerloo, "An approach for ranking of fuzzy numbers," Expert Systems with Applications, vol. 39, no. 1, pp. 690-695, 2012.

[77] H. Akyar, "Fuzzy risk analysis for a production system based on the Nagel point of a triangle," Mathematical Problems in Engineering, Article ID 3080679, 9 pages, 2016.

[78] S. Rezvani, "Ranking generalized exponential trapezoidal fuzzy numbers based on variance," Applied Mathematics and Computation, vol. 262, pp. 191-198, 2015.

[79] Y.-M. Wang, J.-B. Yang, D.-L. Xu, and K.-S. Chin, "On the centroids of fuzzy numbers," Fuzzy Sets and Systems, vol. 157, no. 7, pp. 919-926, 2006.

[80] P. Anand Raj and D. Nagesh Kumar, "Ranking alternatives with fuzzy weights using maximizing set and minimizing set," Fuzzy Sets and Systems, vol. 105, no. 3, pp. 365-375, 1999.

[81] R. Chutia and B. Chutia, "A new method of ranking parametric form of fuzzy numbers using value and ambiguity," Applied Soft Computing Journal, vol. 52, pp. 1154-1168, 2017.

[82] H. Deng, "Comparing and ranking fuzzy numbers using ideal solutions," Applied Mathematical Modelling, vol. 38, no. 5-6, pp. 1638-1646, 2014.

[83] S. Abbasbandy and B. Asady, "Ranking of fuzzy numbers by sign distance," Information Sciences, vol. 176, no. 16, pp. 2405-2416, 2006.

[84] F. Choobineh and H. Li, "An index for ordering fuzzy numbers," Fuzzy Sets and Systems, vol. 54, no. 3, pp. 287-294, 1993.

[85] S. H. Chen, "Ranking fuzzy numbers with maximizing set and minimizing set," Fuzzy Sets and Systems, vol. 17, no. 2, pp. 113129, 1985.

[86] Y. Deng, Z. Zhenfu, and L. Qi, "Ranking fuzzy numbers with an area method using radius of gyration," Computers \& Mathematics with Applications, vol. 51, no. 6-7, pp. 1127-1136, 2006.

[87] B. Asady and A. Zendehnam, "Ranking fuzzy numbers by distance minimization," Applied Mathematical Modelling, vol. 31, no. 11, pp. 2589-2598, 2007.

[88] S.-M. Chen and J.-H. Chen, "Fuzzy risk analysis based on ranking generalized fuzzy numbers with different heights and different spreads," Expert Systems with Applications, vol. 36, no. 3, pp. 6833-6842, 2009.

[89] S.-M. Chen, A. Munif, G.-S. Chen, H.-C. Liu, and B.-C. Kuo, "Fuzzy risk analysis based on ranking generalized fuzzy numbers with different left heights and right heights," Expert Systems with Applications, vol. 39, no. 7, pp. 6320-6334, 2012.

[90] S. H. Nasseri, M. M. Zadeh, M. Kardoost, and E. Behmanesh, "Ranking fuzzy quantities based on the angle of the reference functions," Applied Mathematical Modelling, vol. 37, no. 22, pp. 9230-9241, 2013. 


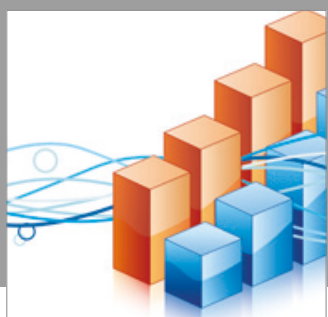

Advances in

Operations Research

vatersals

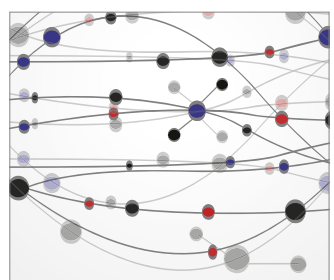

\section{The Scientific} World Journal
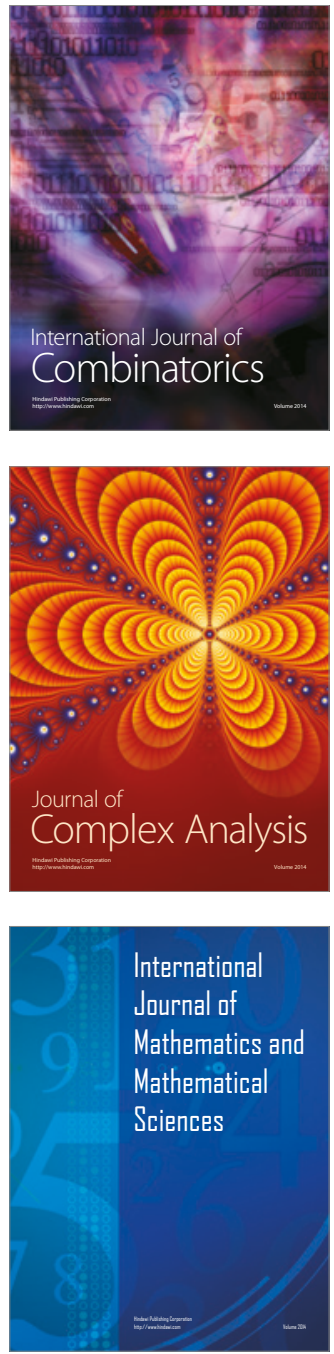
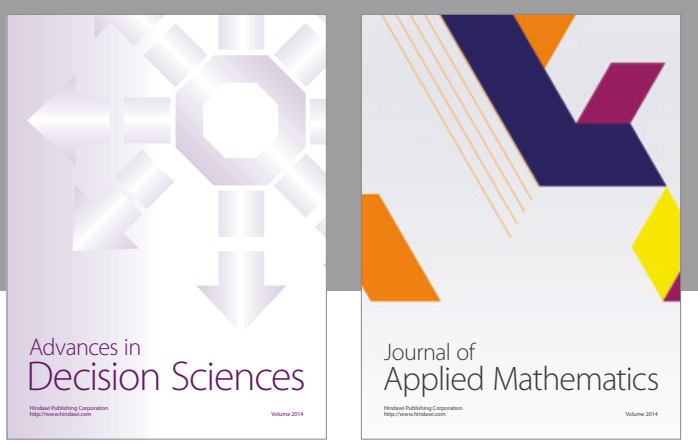

Algebra

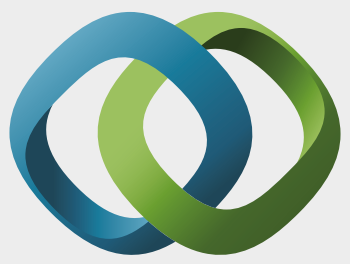

\section{Hindawi}

Submit your manuscripts at

https://www.hindawi.com
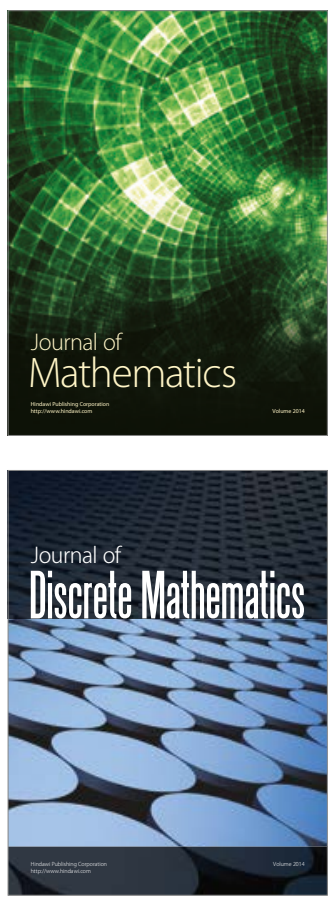

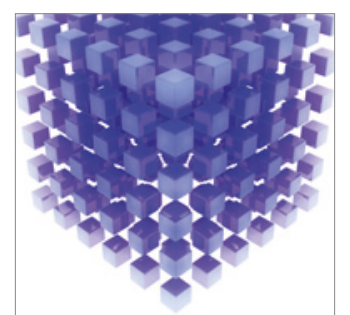

Mathematical Problems in Engineering
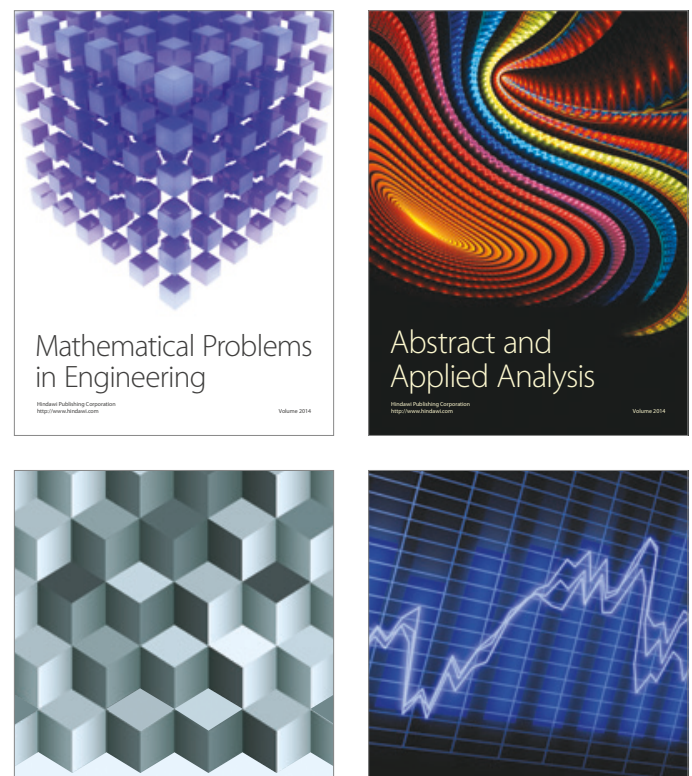

Journal of

Function Spaces

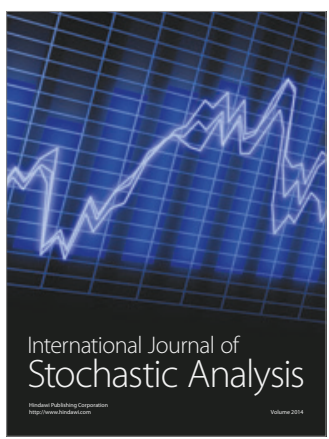

Probability and Statistics
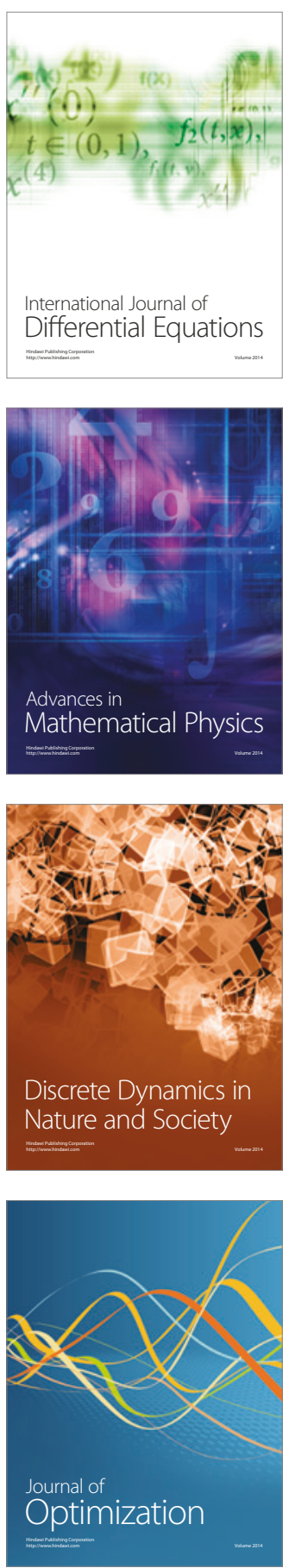